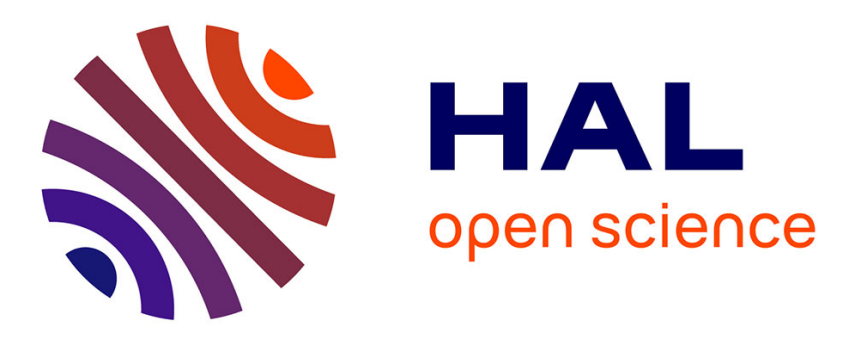

\title{
Les politiques territoriales en Europe de l'Ouest (Allemagne, Espagne, France, Grande-Bretagne, Italie, Communauté européenne)
}

Richard Balme, Philippe Garraud, Vincent Hoffmann-Martinot, Évelyne Ritaine

\section{To cite this version:}

Richard Balme, Philippe Garraud, Vincent Hoffmann-Martinot, Évelyne Ritaine. Les politiques territoriales en Europe de l'Ouest (Allemagne, Espagne, France, Grande-Bretagne, Italie, Communauté européenne). Revue Française de Science Politique, 1993, 43 (3), pp.435-468. 10.3406/rfsp.1993.394753 . halshs-00105561

\section{HAL Id: halshs-00105561 \\ https://shs.hal.science/halshs-00105561}

Submitted on 26 Apr 2019

HAL is a multi-disciplinary open access archive for the deposit and dissemination of scientific research documents, whether they are published or not. The documents may come from teaching and research institutions in France or abroad, or from public or private research centers.
L'archive ouverte pluridisciplinaire HAL, est destinée au dépôt et à la diffusion de documents scientifiques de niveau recherche, publiés ou non, émanant des établissements d'enseignement et de recherche français ou étrangers, des laboratoires publics ou privés. 


\section{Les politiques territoriales en Europe de l'Ouest (Allemagne,}

\section{Espagne, France, Grande-Bretagne, Italie, Communauté}

\section{européenne)}

Monsieur Vincent Hoffmann-Martinot, Monsieur Richard Balme, Monsieur Philippe Garraud, Madame Evelyne Ritaine

\section{Citer ce document / Cite this document :}

Hoffmann-Martinot Vincent, Balme Richard, Garraud Philippe, Ritaine Evelyne. Les politiques territoriales en Europe de l'Ouest (Allemagne, Espagne, France, Grande-Bretagne, Italie, Communauté européenne). In: Revue française de science politique, $43^{\mathrm{e}}$ année, $\mathrm{n}^{\circ} 3,1993$. pp. 435-468;

doi : https://doi.org/10.3406/rfsp.1993.394753

https://www.persee.fr/doc/rfsp_0035-2950_1993_num_43_3_394753

Fichier pdf généré le 25/04/2018 


\title{
Résumé
}

Les politiques territoriales (entendues comme politiques de réforme des gouvernements locaux et intermédiaires, et politiques de réduction des disparités spatiales) ont beaucoup évolué ces dernières années en Europe de l'Ouest. Elles ont donné lieu à une abondante littérature scientifique dont le bilan s'articule autour de trois axes de réflexion. Les débats scientifiques demeurent marqués par la spécificité des enjeux nationaux et par la culture scientifique propre à chaque pays. Une relative convergence apparaît cependant autour de quelques paradigmes centraux : dynamique interorganisationnelle, échange entre intérêts organisés et gouvernements locaux et intermédiaires, territorialisation des cultures poli-tiques, par exemple. Enfin, la construction européenne est partout considérée comme une remise en cause des politiques territoriales nationales : l'intégration économique modifie la distribution spatiale des activités économiques ; l'intégration politique déborde les cadres de l'action publique nationale (notamment par le développement des politiques régionales de la CEE).

\begin{abstract}
Territorial policies in Western Europe

(Germany, Spain, France, Great Britain, ItalY, the EC)

Territorial policies (understood as reform policies applied to local and intermediate government, and policies for reducing spatial disparities) have changed substantially in Western Europe in recent years. Much scientific literature has been produced concerning three Unes of thinking. Scholarly discussions have remained marked by the specificity of national stakes and by the scientific culture proper to each country. A degree of convergence has appeared, however, around a few central paradigms, e.g. the inter-organizational dynamic ; exchanges between organized interests and local and intermediate governments ; and the territorialization of political cultures. Lastly, European construction is considered everywhere as a challenge to national territoral policies : économie integration modifies the spatial distribution of economie activities ; political integration transcends the framework of national public action (particularly through the development of EC regional policies).
\end{abstract}




\title{
LES POLITIQUES TERRITORIALES EN EUROPE DE L'OUEST
}

\author{
Allemagne, Espagne, France, Grande-Bretagne, Italie, \\ et Communauté européenne
}

\section{RICHARD BALME, PHILIPPE GARRAUD, VINCENT HOFFMANN-MARTINOT, EVELYNE RITAINE}

(avec la collaboration de Laurence BONNET et Stéphane LE MAY)

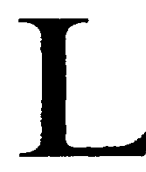

'OBJET de ce bilan* est de rendre compte de la littérature scientifique traitant des politiques territoriales en Europe de l'Ouest au cours de la dernière décennie. On entend par-là les programmes publics dont la finalité est la gestion des territoires dans leurs dimensions politiques et socio-économiques. Une grande partie des interventions des administrations et des gouvernements centraux (en matière d'industrie, d'agriculture, de fiscalité ou de transport, par exemple) ont à l'évidence des implications territoriales. Pour cerner le champ de notre étude, nous avons retenu les politiques les plus explicites en la matière, c'est-à-dire les politiques institutionnelles de réformes des gouvernements locaux ou intermédiaires, et les programmes visant à réduire les disparités spatiales, politiques d'aménagement du territoire, politiques régionales ou politiques de développement, selon la terminologie en vigueur. Ne pouvant prétendre à l'exhaustivité, nous avons centré notre approche sur les cinq grands pays européens, Allemagne, Espagne, France, Italie et Royaume-Uni, auxquels s'ajoute la Communauté européenne dont la construction met en cause l'intégration et dont les politiques affectent de plus en plus ouvertement l'agencement territorial des Etats-membres. Il convient néanmoins de dire ici que ce choix ne sous-estime en rien l'importance de ces problèmes et la vigueur des débats scientifiques qu'ils suscitent dans les autres pays. Le lecteur doit, faute de mieux, être averti de l'importance de la réforme de régionalisation mise en place en Belgique et des réflexions autour de son éventualité au Portugal ou aux Pays-Bas, que les contraintes de cette recherche ne nous permettront pas d'aborder ici.

Le premier de ces deux aspects des politiques territoriales, les caractères institutionnels des gouvernements locaux et leurs réformes, est bien couvert par la littérature ${ }^{1}$. Les politiques d'aménagement ou de déve-

* La recherche dont est tiré ce bilan des travaux a été réalisée grâce à l'appui de la DATAR (Prospective et Territoire). Elle fait l'objet d'une publication (Paris, L'Harmattan, coll. "Logiques politiques ») à paraître fin 1993.

1. Y. Mény, La réforme des collectivités locales en Europe: stratégies et résultats, Paris, La Documentation française, 1984 ; E.C. Page, M.J. Goldsmith, Central and local government relations. A comparative analysis of West European unitary states, Londres, Sage, 1987 ; B. Dente, F. Kjellberg, The dynamics of institutional change. Local government reorganization in Western democracies, Londres, Sage, 1988 ; J. Delcamp, Les institutions locales en Europe, Paris, PUF, 1990. 
loppement sont en revanche peu traitées par l'analyse comparative, et rarement envisagées sous l'angle de leur articulation avec les relations politiques centre-périphérie. Nous espérons donc, en confrontant ces deux problèmes, ouvrir le champ de la réflexion. Par ailleurs, si les contacts internationaux entre les universitaires se développent, l'analyse comparée reste jusqu'ici le plus souvent une juxtaposition d'études empiriques dont les résultats sont malcommodes à synthétiser et les implications théoriques difficiles à percevoir. Il en résulte une sorte d'isolement des spécialistes du gouvernement local au sein de leurs disciplines respectives et, à notre sens, un manque de consolidation des acquis méthodologiques et des résultats. Pourtant, dans chacun des pays retenus, les questions territoriales sont des problèmes politiques majeurs qui ont donné lieu à des travaux d'une grande richesse en science politique et administrative, en sociologie et en économie, et à des traditions d'analyse dont la confrontation nous semble importante à la fois pour progresser dans l'étude des phénomènes considérés et pour dégager l'apport de cet objet à la science politique et aux sciences sociales.

Afin d'avancer dans cette voie, nous examinons particulièrement ici les paradigmes à l'œuvre dans chacun de ces pays, l'ensemble des notions, des concepts et quelquefois des techniques qui organisent le débat scientifique relatif aux politiques territoriales. Nous voudrions faire apparaître leurs spécificités, leurs évolutions et leurs points de convergence. Il fait peu de doute que le contexte culturel et l'objet d'étude donnent sa tonalité à l'interprétation et conditionnent les outils conceptuels des observateurs. On peut à cet égard évoquer la thèse du "pouvoir périphérique " élaborée en France avant la décentralisation, le succès du paradigme inter-organisationnel dans l'interprétation du fédéralisme allemand, la vitalité de la production d'inspiration marxiste dans la Grande-Bretagne de Margaret Thatcher où le gouvernement local devient un enjeu idéologique, ou encore le raisonnement en termes de «fragmentation " suscitée par l'analyse du système politique italien. Il est néanmoins possible de déceler des échanges, des influences mutuelles et des points d'accord entre l'ensemble de ces analyses. Nous voudrions tenter de les expliciter, même s'il est clair que l'ampleur du sujet exigerait des développements plus détaillés et plus nuancés, et que les essais de généralisation à partir d'une littérature aussi vaste et diversifiée exposent au risque d'une réduction. L'exercice mérite d'être tenté, mais il impose la prise en considération d'un nombre important de travaux dont nous proposerons par conséquent dans les pages suivantes davantage une synthèse qu'une critique approfondie.

En ce qui concerne les paradigmes utilisés, on peut d'abord déceler un affaiblissement des clivages scientifiques dans chacun des pays, à l'exception peut-être de la Grande-Bretagne où la tendance est moins nette. Comme dans l'ensemble de la science politique, les affrontements d'écoles entre pluralistes, marxistes et institutionnalistes actifs dans les années 1970, sans disparaître complètement, se sont sensiblement estompés. On peut y voir les avantages d'un consensus plus fort entre les analystes et des capacités de dialogue entre des perspectives différentes, 
et donc des interprétations plus fines, plus nuancées. On peut aussi regretter une forme de recul théorique cédant le pas à une fuite en avant empirique, au mieux étayée de "théories intermédiaires » dont l'intérêt plus général pour la connaissance des phénomènes politiques est peu évident. Pourtant, un certain nombre de récurrences apparaissent sous forme fragmentée dans les problématiques et les cadres conceptuels, donnant tout leur intérêt à ces recherches.

En premier lieu, beaucoup de ces travaux sont directement issus ou sont fortement influencés par la sociologie des organisations, et appliquent à l'étude des relations inter-gouvernementales les notions de l'analyse inter-organisationnelle, en raisonnant, notamment, en termes de rationalité limitée, de systèmes d'action et de réseaux. Ce courant a connu des développements importants en France et en Allemagne, puis en Grande-Bretagne ${ }^{1}$, et apporte une contribution importante, par son application aux relations centre-périphérie, à la sociologie des organisations. De manière explicite ou implicite, il forme sans doute le point de consensus le plus fort entre les auteurs. Le deuxième champ problématique récurrent est celui des rapports entre les gouvernements locaux et les intérêts organisés, dont la version la plus théoriquement intégrée est le débat sur le corporatisme, envisagé au niveau local mais aussi au niveau plus globalement européen ${ }^{2}$. Il s'agit d'analyser le rôle des gouvernements locaux et intermédiaires dans l'articulation des intérêts, et les nouvelles régulations assurées par les politiques publiques face à la recomposition des échanges économiques et ses incidences territoriales. Même si l'interprétation ne suscite pas le même consensus et reste au contraire assez nettement partagée entre les adeptes et les sceptiques, c'est cette notion de corporatisme qui structure le débat et qui souligne l'importance de la question de la régulation des intérêts dans les analyses envisagées. Enfin, ces travaux considèrent de plus en plus précisément les déterminants locaux des politiques, notamment l'influence des cultures politiques locales sur le leadership et les performances institutionnelles dans la mise en cuvre des programmes publics. Le concept de "néolocalisme », forgé en Italie, illustre parfaitement cette démarche ${ }^{3}$ et trouve des correspondances significatives dans les autres pays. C'est probablement l'intégration entre ces trois problématiques et les concepts qu'elles privilégient, qui permettra à l'avenir de progresser dans la compréhension des politiques territoriales.

1. M. Crozier, J.-C. Thoenig, «La régulation des systèmes organisés complexes. Le cas du système de décision politico administratif local en France », Revue française de sociologie, 16, 1975, p. 3-32; F.W. Sharpf, B. Reissert, F. Schnabel, Politikverflechtung: Theorie und Empirie des Kooperativen Föderalismus in der Bundesrepublik, Kronberg Ts., Scriptor Verlag, 1976 (Monographien Ergebnisse der Sozialwissenschaften, 1) ; K. Hanf, F.W. Sharpf, Interorganizational policy making. Limits to coordination and central control, Londres, Sage, 1978 ; R.W. Rhodes, The national world of local government, Londres, Allen and Unwin, 1986.

2. W. Streeck, P.C. Schmitter, «From national corporatism to transnational pluralism : organized interests in the Single European Market », Politics and Society, 19 (2), 1991 , p. 133-164.

3. C. Trigilia, «La regolazione localistica: economia e politica nelle aree di piccola impresa », Stato e Mercato, 14, 1985. 
Richard Balme et al.

En termes d'interprétation cette fois, quelles sont les conclusions générales établies par cette littérature? D'abord, le constat d'une convergence partielle dans les pays considérés dans les relations institutionnelles entre le centre et la périphérie. Les politiques de décentralisation dans les Etats fortement centralisés (France et Espagne), de centralisation en Grande-Bretagne où l'autonomie du gouvernement local était traditionnellement forte, l'imbrication croissante des politiques et des niveaux de gouvernement en Allemagne et les progrès de la régionalisation italienne concordent pour développer l'intégration entre le centre et la périphérie. Les gouvernements locaux ou intermédiaires gagnent en autonomie là où ils étaient les plus dépendants, et voient se développer leur interdépendance avec le centre là où ils étaient les plus autonomes. Cette convergence n'est qu'une tendance limitée d'évolution qui n'annonce en aucun cas une disparition des différences et des spécificités dans un avenir envisageable, notamment parce qu'une ligne de clivage sépare encore nettement les Etats unitaires et les systèmes fédéraux ou semi-fédéraux. Mais cette évolution est nettement sensible, parfois spectaculaire, comme dans le cas de l'Espagne. Témoignant de cette interdépendance accrue, les observateurs notent la juridicisation ou tout au moins le développement d'un contentieux juridique entre l'Etat et les gouvernements locaux, qui reflète l'élargissement des transactions et des négociations entre niveaux de gouvernement.

Malgré cet aspect institutionnel des politiques territoriales, on enregistre dans l'ensemble des pays un retrait des politiques régionales ou des politiques d'aménagement du territoire, 'qui n'est que partiellement supplanté par les politiques de la CEE. L'aménagement planifié ou les politiques régionales cèdent le pas à une conception du développement local plus endogène et recourant davantage à des intervenants privés. La libéralisation des politiques économiques et le retrait des politiques régionales coïncident avec l'activation de tensions territoriales ${ }^{1}$, notamment dans les périphéries où elles peuvent donner lieu à une résurgence ou une nouvelle construction des mouvements autonomistes ou séparatistes ${ }^{2}$. Dans cette perspective, les chercheurs sont le plus souvent conduits à s'interroger sur la pertinence et sur les performances du niveau régional dans ces problèmes d'intégration politique.

Ces travaux dégagent également l'expansion de ce qu'on peut appeler les relations inter-gouvernementales élargies, c'est-à-dire les relations transversales de coopération entre niveaux de gouvernement. Ces relations entre gouvernements locaux ont une dimension locale, contiguë, de plus en plus fréquente dans les politiques publiques, et une dimension délocalisée, parfois trans-nationale, celle de la coopération décentralisée, dont les implications, notamment pour la construction communautaire, sont encore difficiles à cerner. Enfin, la question de la participation des citoyens au niveau local nourrit une part importante de la littérature,

1. R.W. Rhodes, V.Wright, Tensions in the territorial politics of Western Europe, Londres, Franck Cass, 1987.

2. M. Keating, State and regional nationalism. Territorial politics and the European state, Hemel-Hampstead, Harvester-Wheatsheaf, 1988. 
que ce soit, comme en Allemagne, pour dégager son influence sur les politiques locales, ou, comme ailleurs, pour comprendre le déficit démocratique dont souffre l'Etat-providence contemporain. En focalisant son investigation sur la dynamique des territoires, sur leur construction et leur traitement politique, ce champ de recherche apporte donc un éclairage indispensable sur les fonctions d'intégration sociale de l'Etat, sur ses contradictions et sur les facteurs qui déterminent son évolution la plus récente.

\section{LE GOUVERNEMENT LOCAL ET LE NOUVEL ÉTAT BRITANNIQUE}

La question de l'autonomie du gouvernement local s'est trouvée placée au centre de l'agenda politique britannique dès le milieu des années 1970. Avec l'arrivée au pouvoir des conservateurs, en 1979, s'ouvre cependant une période de crise dans les relations central-local, nettement plus problématiques. Plusieurs axes de recherche se sont alors développés, restructurant le champ du débat scientifique. L'opposition marxiste-pluraliste se perpétue autour de l'étude des processus d'élaboration des politiques au niveau local. De nombreux travaux sont consacrés à la nature des systèmes politiques locaux. Parallèlement, des perspectives théoriques plus ambitieuses se sont centrées sur l'analyse des relations inter-gouvernementales élargies. Distinctes dans leurs démarches respectives, ces approches élaborent une théorie globale des relations central-local. L'étude des politiques territoriales fournit enfin un domaine de recherche en expansion, notamment sous l'effet de la régionalisation et de la question des tensions territoriales dans les périphéries nationales.

\section{LA REDÉCOUVERTE DU GOUVERNEMENT LOCAL}

Le débat sur la nature du gouvernement local a été significativement renouvelé (après l'approche marxiste de C. Cockburn ${ }^{1}$ ) par la perspective néo-marxiste de la thèse de l'Etat dual (Dual State Thesis) de P. Saunders ${ }^{2}$. L'intervention étatique au niveau central serait essentiellement liée au processus de production, alors que "l'Etat local » assurerait une activité dirigée prioritairement vers la consommation. Au niveau central, les intérêts de classe attenant à la production sont régulés par une médiation reposant sur des arrangements corporatistes, alors qu'au plan local la consommation donne lieu à des luttes d'intérêts concurrentiels de forme pluraliste. Critiquée notamment pour la difficulté à soutenir une telle ligne de partage entre niveaux de politiques et aires

1. C. Cokburn, The local state. Management of cities and people, Londres, Pluto Press, 1978.

2. P. Saunders, Urban politics : a sociological approach, Londres, Hutchinson, 1979. 


\section{Richard Balme et al.}

d'activités ${ }^{1}$, la thèse de l'Etat dual a cherché à intégrer la nouvelle donne des relations central-local: tentatives de réduction des dépenses des autorités locales; remodelage de leurs compétences; accent mis sur le rôle du secteur privé. La restructuration du gouvernement local pourrait s'analyser comme part d'un changement plus large, celui du Welfare State, se traduisant par la représentation et l'influence accrues des intérêts professionnels au niveau local ${ }^{2}$. L'assouplissement du modèle théorique initial peut être apprécié par les nombreuses études relatives à l'éventuelle émergence de modes corporatistes de médiation au niveau local $^{3}$, dont le bilan semble néanmoins à nuancer ${ }^{4}$.

\section{L'ÉTUDE DES RELATIONS INTER-GOUVERNEMENTALES ÉLARGIES}

«La politique locale est restée plus séparée de la politique nationale que dans presque aucun autre pays », écrit J. Bulpitt pour signaler la singularité britannique décrite par le modèle de la dual polity ${ }^{5}$. Avec les réformes, les terrains d'accès entre le centre et la périphérie se sont déplacés, et de nouveaux canaux d'interaction sont apparus. Martin Loughlin $^{6}$ décrit la politisation et la juridicisation de ces relations. Le « lien partisan» s'est trouvé élargi, modifiant l'isolation traditionnelle des sphères politiques nationale et locale ${ }^{7}$, alors que les canaux d'accès central-local se sont déplacés sur le terrain des tribunaux. Pour dépasser les limites d'un modèle axé sur une perspective trop fonctionnaliste comme la thèse de l'Etat dual, on a pu adopter une approche proprement focalisée sur les processus d'élaboration des politiques, pour caractériser le maillage national-local des réseaux de politiques (policy networks) ${ }^{8}$. Les relations central-local sont dès lors abordées sous l'angle des réseaux sectoriels et de l'impact différencié des mesures centralisatrices sur ceux$\mathrm{ci}^{9}$.

Partant du constat de l'échec des travaux sur l'« Etat local » à rendre compte de la crise actuelle des relations central-périphérie, S. Duncan et

1. P. Dunleavy, Urban political analysis, Londres, Macmillan, 1980.

2. P. Saunders, "Rethinking local politics», dans M. Boddy, C. Fudge (eds), Local socialism? Labour councils and new left alternatives, Londres, Macmillan, 1984, p. 22-48.

3. J. Dearlove, The reorganisation of British local government, Cambridge, Cambridge University Press, 1979; I. Holliday, "The new suburban right in British local government: conservative views of the local », contribution à la Political Studies Association Conference, Durham, 1990.

4. A. Cochrane, "The changing state of local government: restructuring for the 1990's ", Public Administration, 4, 1991, p. 281-302; A. Harding, "The crisis of urban growth coalitions, UK style ?», Government and Policy, 9 (3), 1991, p. 295-318.

5. J. Bulpitt, Territory and power in the UK: an interpretation, Manchester, Manchester University Press, 1983 ; M.C. Goldsmith, E.C. Page, "Britain in central and local government relations ", dans Central and local government relations: a comparative analysis of West unitary states, Londres, Sage, 1987 (Sage Modern Politic Series). 1986.

6. M. Loughlin, Local government and the modern State, Londres, Sweet and Maxwell, 1983.

7. J. Gyford, M. James, National parties and local politics, Londres, Allen and Unwin,

8. R.W. Rhodes, The national world..., op. cit.; R.W. Rhodes, « Policy networks : a British perspective ", Journal of Theoretical Politics, 2 (3), 1990, p. 291-318.

9. G. Stoker, The politics of local government, Londres, Macmillan, 1988. 
M. Goodwin introduisent une perspective historique marquée dans leur analyse néo-marxiste. Des tensions se sont déjà exprimées dans le passé (Poplarism dans les années 1920 ou Clay Cross dans les années 1970). Elles ne font que traduire la double nature contradictoire de l'Etat local, à la fois représentant du pouvoir central et interprète de celui-ci au niveau local, et capable d'entreprendre des politiques alternatives crédibles ${ }^{1}$.

\section{POLITIQUES RÉGIONALES ET AUTONOMIE LOCALE}

On rappellera ici brièvement les analyses de la politique régionale (résiduelle par bien des aspects) avant d'examiner l'enjeu représenté par l'instauration d'un niveau régional d'élaboration des politiques. Le maintien d'une politique régionale minimale peut s'expliquer, outre par la nécessité de capter les subventions de la CEE, par la pression politique des partis cherchant à paraître actifs dans ce domaine ${ }^{2}$. Ses limites sont sans doute liées à la menace représentée par la constitution d'une telle sphère de différenciation politique pour le système bipartisan britannique, et la politique régionale poursuivie peut être analysée comme un instrument de régulation de cette menace ${ }^{3}$.

Plus nettement que la lutte contre les disparités spatiales, l'affirmation d'une administration de niveau régional a relancé l'étude des politiques publiques territoriales. L'Etat régional permet de transférer des compétences vers des instances non élues ${ }^{4}$. La régionalisation, selon le gouvernement Thatcher, ne serait ainsi qu'une «variante de la nationalisation de l'autonomie locale ${ }^{5}$.

Un autre axe dans l'analyse des politiques publiques territoriales a été celui du territorial management, c'est-à-dire de la façon dont le gouvernement central gère ses périphéries «nationales». Outre les Offices $^{6}$, les Agences de développement régional se présentent comme un moyen pour le centre de gérer des périphéries où les intérêts régionaux sont bien articulés, en évitant la mise à disposition directe de ressources aux autorités locales. Les tensions territoriales peuvent néanmoins perdurer et s'activer sous de nouvelles formes porteuses de modernité : à la

1. S. Duncan, M. Goodwin, The local State and uneven development: behind the local government crisis, Oxford, Polity Press, 1988 ; S. Duncan, M. Goodwin, «Uneven development and local social relations ", International Journal of Urban and Regional Research, 12 (1), 1988, p. 107-129.

2. P.J. Damesick, P. Wood, Regional problems, problems regions and public policy in the United Kingdom, Oxford, Clarendon Press, 1987.

3. I.R. Gordon, "Regional policy and national politics», Government and Policy, 8 (4), 1990 , p. $427-438$.

4. J. Dearlove, P. Saunders, Introduction to British politics, Oxford, Polity Press, 1984.

5. S. Duncan, M. Goodwin, The local State..., op. cit.

6. M.C. Goldsmith, "Managing the periphery in a period of fiscal stress », dans M.C. Goldsmith, New research in central-local relations, Aldershot, Gower, 1986 ; J.G. Kellas, "The Scottish and Welsh offices as territorial managers", Regional Politics and Policy, 1 (1), 1991, p. 87-100; R. Parry, "Sub-national bureaucracy in the United Kingdom: the Scottish office in the 1980s ", dans R. Balme, V. Hoffmann-Martinot, Local and regional bureaucracies in Western Europe, Talence, Institut d'études politiques de Bordeaux, 1991, p. 109-124 («Les cahiers du CERVL », n²). 
Richard Balme et al.

résistance culturelle traditionaliste à l'intégration à l'Etat-nation viendraient s'ajouter les mobilisations et négociations dans les politiques publiques, qui définissent les nouveaux rapports centre-périphérie ${ }^{1}$.

La place du gouvernement local s'est donc retrouvée au centre de l'agenda politique comme du débat scientifique pendant les années du gouvernement Thatcher. L'étude des relations centre-périphérie a nourri en Grande-Bretagne différentes analyses porteuses d'une théorie globale de l'Etat et a été un point de restructuration du champ académique. Le foisonnement et la richesse de ces travaux constituent, enfin, l'une des marques importantes de la résurgence de la question de la territorialité en science politique ${ }^{2}$.

\section{ALLEMAGNE : À LA RECHERCHE DES DÉTERMINANTS LOCAUX DES POLITIQUES TERRITORIALES}

Si on la mesure à l'aune du nombre des publications qui se sont multipliées depuis une vingtaine d'années, l'analyse des phénomènes politiques territorialisés apparaît comme l'une des branches les plus dynamiques de la science politique allemande contemporaine. Pourtant, jusque dans les années 1970, les études locales n'intéressaient guère que les juristes et rarement les politistes. La "découverte », à partir de la fin des années 1960, de la scène politique locale s'est effectuée principalement à travers le suivi et les tentatives d'interprétation des effets de la transformation des relations inter-gouvernementales, d'une part, et des politiques de rénovation urbaine, d'autre part. Depuis les premières années de tâtonnement marquées davantage par des affirmations et oppositions idéologiques - entre, notamment, pluralistes et marxistes que par de réelles avancées théoriques et méthodologiques, ce champ de recherche n'a cessé de se structurer et de se différencier. Parallèlement aux recherches portant sur l'analyse des systèmes politiques locaux et régionaux, se sont progressivement développés des travaux consacrés, d'une part, à la dynamique des relations inter-gouvernementales et, plus récemment, à l'analyse des politiques publiques territoriales.

\section{L'ANALYSE DES SYSTĖMES POLITIQUES LOCAUX ET RÉGIONAUX}

On a assisté en Allemagne depuis la fin des années 1960 à une rénovation de la scène politique locale, qui a considérablement dynamisé la gestion des collectivités territoriales. Cette légitimation accrue du local a été portée par une mobilisation sans précédent de citoyens organisés en «initiatives» (Bürgerinitiativen) et multiples associations, relayées politiquement par le mouvement des Grüne (les Verts), devenu dans les

1. M. Keating, State and regional nationalism, op. cit.

2. R.W. Rhodes, V. Wright, Tensions..., op. cit. 
années 1980 le parti-phare de l'écologisme européen. Cette profonde transformation du paysage politique ouest-allemand a été directement à l'origine de la relocalisation du discours, des pratiques et stratégies des organisations partisanes, investissant de plus en plus le champ politique local.

Ce phénomène de montée en puissance des systèmes socio-politiques locaux est au centre de l'ouvrage collectif édité par J.J. Hesse ${ }^{1}$ autour de trois thèmes principaux : la modification du système partisan traditionnel provoquée par l'émergence de nouveaux mouvements sociaux et des Grüne; l'engorgement et le blocage du système fédéral provoqués par la juridicisation et la bureaucratisation de la société, ainsi que par des mouvements en sens inverse de décentralisation et de régionalisation; la transformation des structures économiques et socio-culturelles conduisant à une revalorisation de la scène locale. Cette thèse est développée dans des travaux plus récents, tels que ceux consacrés aux formes nouvelles de régulation politique ${ }^{2}$, aux pratiques des nouveaux mouvements sociaux urbains incarnant de nouveaux clivages se substituant à celui longtemps dominant de classe ${ }^{3}$, ou aux politiques innovantes, menées dans les villes cogérées par les Grüne ${ }^{4}$. Relocalisation englobe également rerégionalisation, au sens où, à l'intérieur de Länder, comme la Basse-Saxe ou la Rhénanie du Nord-Westphalie, les régions, qui n'ont pas en Allemagne de physionomie institutionnelle aussi clairement délimitée que leurs homologues italiennes ou françaises, se voient attribuer de plus en plus de prérogatives afin notamment de renforcer l'effectivité des politiques étatiques: il n'y aurait donc pas un mouvement exclusif vers la centralisation ou au contraire la décentralisation, mais concomitance de ces tendances. Les régions deviennent des acteurs charnières faisant le pont entre le secteur privé, l'Etat et les communes, si bien que D. Fürst évoque "l'écologisation des politiques sectorielles »" 5 .

Cette tendance lourde à la décentralisation des activités publiques profite-t-elle autant au citoyen qu'à ses représentants, élus ou fonctionnaires? Cette question est au cœur de l'évaluation de la vaste réforme territoriale et fonctionnelle des années 1960. En l'espace de dix ans, le nombre des communes fut divisé par trois et celui des Kreise environ

1. J.J. Hesse, H. Gänseforth, D. Fürst, E.H. Ritter (Hrsg.), Staat und Gemeinden zwischen Konflikt und Kooperation, Baden-Baden, Nomos, 1983.

2. U. Bullmann, "Zur "Identität der lokalen Ebene". Aussichten zwischen kommunaler Praxis und politikwissenschaftlicher Theorie ", dans Bernhard Blanke (Hrsg.), Staat und Stadt. Systematische, vergleichende und problemorientierte Analysen "dezentraler Politik», Opladen, Westdeutscher Verlag, 1991 (Politische Vierteljahresschrift, Sonderheft 22).

3. P. Grottian, W. Nelles (Hrsg.), Großstadt und neue soziale Bewegungen. Bâle, Boston, Stuttgart, Birkhäuser Verlag, 1983 (Stadtforschung Aktuell, Bd. 1).

4. U. Bullmann, P. Gitschmann, P. Henkenborg, «Zwischenbilanz rot-grüner Politik in den hessischen Kommunen ", dans Roland Roth, Hellmut Wollmann (Hrsg.), Grünalternative Kommunalpolitik in der Bundesrepublik-eine Zwischenbilanz, Bâle, Boston, Stuttgart, Birkhaüser Verlag, 1991 (Stadtforschung Aktuell); R. Roth, "Local Green politics in West German cities », International Joumal of Urban and Regional Research, 15 (1), mars 1991 , p. $75-89$.

5. D. Fürst, W. Klinger, J. Knieling, M. Mönnecke, H. Zeck, Regionalverbände im Vergleich, Baden-Baden, Nomos, 1990 (Schriften zur kommunalen Wissenschaft und Praxis, Bd. 4). 
Richard Balme et al.

par deux. L. Albertin et ses associés ${ }^{1}$ ont dressé un bilan de cette réforme dans la région de Westphalie orientale-Lippe, soulignant son déficit démocratique; d'inspiration essentiellement technocratique, son objectif central était de renforcer les appareils administratifs existants, et très accessoirement de consolider les structures de représentation démocratiqué. Décentralisation ne rime pas nécessairement avec démocratisation, loin s'en faut, ainsi que l'illustrent les nombreuses monographies approfondies sur lesquelles s'appuient aussi bien W. Bückmann et H.U. Oel ${ }^{2}$ que G. Wehland ${ }^{3}$. Sur les différentes dimensions et formes institutionnelles et politiques de la participation du citoyen à la politique locale, on se reportera plus généralement aux deux précieux ouvrages collectifs dirigés par Oscar W. Gabriel ${ }^{4}$. Ces recherches allemandes sur les systèmes politiques locaux témoignent d'une belle variété de méthodes utilisées, allant de l'analyse patiente et fouillée de l'évolution des mécanismes du pouvoir local dans une ville ${ }^{5}$ à la comparaison inter-municipale basée sur une vaste enquête par questionnaire du profil et des pratiques des 1090 maires du Bade-Wurtemberg ${ }^{6}$, en passant par la mise en perspective de la place et des stratégies des partis d'opposition dans les dix plus grandes villes de Rhénanie du Nord-Westphalie ${ }^{7}$.

\section{LA DYNAMIQUE DES RELATIONS INTER-GOUVERNEMENTALES}

L'analyse moderne des systèmes fédéraux a relativisé l'importance attribuée à l'exercice séparé des fonctions, pour mettre davantage en évidence les mécanismes et contraintes des interactions entre la Fédération et les Etats, bref la dynamique du fédéralisme. Les scientifiques et praticiens ouest-allemands ont importé des Etats-Unis la notion de «fédéralisme coopératif », afin d'exprimer et d'étudier l'institutionnalisation des relations conjuguant l'action du Bund, des Länder et des collectivités territoriales. Approfondissant cette voie fructueuse, Fritz W. Scharpf et ses associés ${ }^{8}$ ont popularisé la notion plus riche " $\mathrm{d}^{\prime i m-}$

1. L. Albertin, K. Eris, R. Werle, Die Zukunft der Gemeinden in der Hand ihrer Reformer. Geplante Erfolge und politische Kosten der kommunalen Neugliederung. Fallstudien in Ostwestfalen-Lippe. Opladen, Westdeutscher Verlag, 1982 (Forschungsberichte des Landes Nordrhein-Westfalen, Fachgruppe Wirtschafts- und Sozialwissenschaften, $\mathrm{n}^{\circ} 3124$ ).

2. W. Bückmann, H.U. Oel, Bürgerbeteiligung bei kommunalen Planungen, Munich, Minerva, 1981 (Beiträge des Instituts für Zukunftsforschung, 20).

3. G. Wehland, Stadtplanung, Partizipation und kommunale Öffentlichkeit. Zum politischen Stellenwert von bürgerschaftlicher Mitwirkung im Bauleitplanverfahren, Berlin, Arbeitshefte des Instituts für Stadt- und Regionalplanung der Technischen Universität Berlin, Heft 30, 1984.

4. O.W. Gabriel (Hrsg.), Bürgerbeteiligung und kommunale Demokratie, Munich, Minerva, 1983 (Beiträge zur Kommunalwissenschaft, Bd. 13) ; O.W. Gabriel (Hrsg.), Kommunale Demokratie zwischen Politik und Verwaltung, Munich, Minerva, 1989 (Beiträge zur Kommunalwissenschaft, Bd. 29).

5. T. Ellwein, R. Zoll, Wertheim. Politik und Machtstruktur einer deutschen Stadt, Munich, Juventa, 1982 (Reihe Politisches Verhalten, Bd. 9).

6. H.G. Wehling, H. Jorg Siewert, Der Bürgermeister in Baden-Württemberg. Eine Monographie, Stuttgart, Kohlhammer, 1984.

7. O.W. Gabriel, P. Haungs, M. Zender, Opposition in Großstadtparlamenten, Melle, Knoth, 1984 (Forschungsbericht/Konrad Adenauer Stiftung, 42).

8. F.W. Scharpf, B. Reissert, F. Schnabel, Politikverflechtung..., op. cit. 


\section{Les politiques territoriales en Europe de l'Ouest}

brication de politiques " (Politikverflechtung) qui donne sens aux interrelations croissantes, institutionnalisées ou non, entre communes, Länder et Bund. L'interprétation en termes de Politikverflechtung, initialement appliquée à l'interdépendance des politiques du Bund et des Länder, a été par la suite étendue aux interactions spécifiques entre échelon municipal et niveaux territoriaux supérieurs. Que les communes soient confrontées dans un système fédéral non pas à un mais à deux niveaux étatiques ne signifie pas que leur autonomie soit doublement encadrée et menacée. On peut soutenir au contraire que cette double structuration favorise leurs stratégies en leur offrant, en fonction des circonstances, des possibilités d'alliances à géométrie variable. Selon les secteurs et politiques analysés, les acteurs étatiques soit parviennent tout de même à renforcer leur contrôle sur les collectivités territoriales ${ }^{1}$, soit demeurent soumis aux pressions multiples de leurs partenaires municipaux et de leurs Spitzenverbände ${ }^{2}$.

Le processus d'imbrication verticale des responsabilités, ressources et actions des acteurs publics territoriaux semble s'accentuer au fil des années. Dans un important essai, F.W. Scharpf ${ }^{3}$ s'emploie d'ailleurs à $^{\prime}$ retracer et à comprendre la convergence des manifestations de blocage des institutions de la CEE, et des tendances à l'immobilisme du système fédéral allemand. Mais c'est aussi horizontalement que le phénomène s'amplifie, l'intercommunalité se développant en Allemagne comme dans d'autres pays industrialisés au rythme de l'intensification des enjeux qu'affrontent aujourd'hui les grandes agglomérations. Parmi les études relativement nombreuses publiées ces dernières années sur cette question clé des territoires européens ${ }^{4}$, on recommandera plus particulièrement la lecture de trois recherches consacrées aux conditions institutionnelles et socio-politiques de l'efficience de la coopération intercommunale et régionale ${ }^{5}$.

1. H. Krischmann, Der Einfluß staatlicher Raumplanung auf die kommunale Planungshoheit. Die Regionalplanung Nordhessen im Verhältnis zu den Bauleitplanungen der Städte Kassel und Baunatal, Munich, Minerva, 1983 (Beiträge zur Kommunalwissenschaft, Bd. 10); $\mathrm{R}$. Quentmeier, Regionalplanung im Planungsverbund. Zur Funktion des Bezirksplanungsrates im Rahmen der Politikverflechtung, Munich, Minerva, 1983 (Beiträge zur Kommunalwissenschaft, Bd. 9).

2. J.J. Hesse et al., Staat und Gemeinden..., op. cit.; D. Fürst, J.J. Hesse, H. Richter (Hrsg.), Stadt und Staat. Verdichtungsräume im Prozeß der föderalstaatlichen Problemverarbeitung, Baden-Baden, Nomos, 1984; W. Jaedicke, K. Ruhland, U. Wachendorfer, H. Wollmann, H. Wonneberg, Lokale Politik im Wohlfahrtsstaat. Zur Sozialpolitik der Gemeinden und ihrer Verbände in der Beschäftigungskrise, Opladen, Westdeutscher Verlag, 1991 (Schriften des Zentralinstituts für sozialwissenschaftliche Forschung der Freien Universität Berlin, Bd. 61).

3. F.W. Scharpf, «The joint-decision trap. Lessons from German federalism and European integration », Public Administration, 66, automne 1988, p. 239-278 (édition revue et -augmentée de "Die Politikverflechtungs-Falle: Europäische Integration und deutscher Föderalismus im Vergleich ", Politische Vierteljahresschrift, 26 (4), décembre 1985, p. 323 356).

4. Voir, entre autres, F. Vorholz, Interkommunale Einrichtungen. Ein Beitrag zur Stadt-Umland-Problematik, Munich, Minerva, 1983 (Beiträge zur Kommunalwissenschaft, Bd. 1).

5. R. Rapior, Interkommunale Zusammenarbeit. Eine empirische Untersuchung über die Stadt-Umland-Beziehungen in der Stadtregion Freiburg, Munich, Minerva, 1984 (Beiträge zur Kommunalwissenschaft, Bd. 15); A. Benz, Regionalplanung in der Bundesrepublik Deutsch- 
C'est précisément le degré élevé d'imbrication des niveaux territoriaux en Allemagne qui a contribué à stimuler davantage, et plus tôt que dans des Etats unitaires comme la France, les tentatives d'analyse des politiques publiques territoriales. Il y eut d'abord la parution d'ouvrages fondamentaux comme celui sur l'implémentation, dirigé par Renate Mayntz $^{1}$ et ceux de G.M. Hellstern et H. Wollmann ${ }^{2}$ qui ont contribué à introduire l'évaluation comme objet d'analyse des politiques publiques en RFA. A suivi une grande variété de productions, très ciblées et généralement consacrées à des politiques sectorielles. Il faut souligner que la plupart de leurs auteurs reconnaissent désormais aux systèmes politiques territoriaux, qu'ils soient municipaux ou régionaux, une autonomie d'action plus réelle que la Politikwissenschaft ou les Kommunalwissenschaften ne l'avaient longtemps (pré)supposé. Grâce à ce changement de paradigme (de la domination-hégémonie des forces nationales-étatiques au néo-localisme), on essaie alors de répondre à des questions cruciales. Les politiques publiques se différencient-elles en fonction de l'idéologie partisane des collectivités territoriales qui les portent (does politics matter?) ? Plusieurs années après le travail pionnier mais isolé de l'américain Fried, cette problématique inspire de nombreuses analyses ${ }^{3}$. Quel est l'impact de la distribution interne des ressources institutionnelles et politiques (plus ou moins de centralisation $\mathrm{du}$ leadership exécutif) sur les policy outputs? Plus précisément, les villes de Bavière et du Bade-Wurtemberg sont-elles plus riches et mieux gérées que celles du Nord non seulement en raison de leur environnement économique, mais aussi parce qu'elles sont dirigées par des maires forts ${ }^{4}$ ? Comment les collectivités locales interviennent-elles territoriale-

land. Eine empirische Untersuchung zur Organisation und Problemlösungsfähigkeit, Münster, Selbstverlag des Instituts für Siedlungs- und Wohnungswesen und des Zentralinstituts für Raumplanung der Universität Münster, 1982 (Beiträge zum Siedlungs- und Wohnungswesen und zur Raumplanung, Bd. 80); D. Fürst et al., Regionalverbände..., op. cit.

1. R. Mayntz (Hrsg.), Implementation politischer Programme II. Ansätze zur Theoriebildung, Opladen, Westdeutscher Verlag, 1983.

2. En particulier, G.M. Hellstern, H. Wollmann (Hrsg.), Evaluierung und Erfolgskontrolle in Kommunalpolitik und -verwaltung, Bâle, Boston, Stuttgart, Birkhäuser Verlag, 1984 (Stadtforschung Aktuell, Bd. 6).

3. O.W. Gabriel, V. Kunz, T. Zapf-Schramm, Bestimmungsfaktoren des kommunalen Investitionsverhaltens, Munich, Minerva, 1990; H. Grüner, W. Jaedicke, K. Ruhland, « Rote Politik im schwarzen Rathaus? Bestimmungsfaktoren der wohnungspolitischen Ausgaben bundesdeutscher Großstädte », Politische Vierteljahresschrift, 29 (1), 1988, p. 42-57; W. Jaedicke et al., Lokale Politik..., op. cit.

4. G. Banner, "Kommunale Steuerung zwischen Gemeindeordnung und Parteipolitik am Beispiel der Haushaltspolitik », Die Offentliche Verwaltung, 1984, p. 364-372; Uwe Winkler-Haupt, «Die Auswirkungen unterschiedlicher kommunaler Führungsorganisationstypen auf den Policy-Output $»$, dans Dieter Schimanke (Hrsg.), Stadtdirektor oder Bürgermeister. Beiträge zu einer aktuellen Kontroverse, Bâle, Boston, Stuttgart, Birkhäuser Verlag, 1989 (Stadtforschung Aktuell, Bd. 23); K. Volker, T. Zapf-Schramm, "Ergebnisse der Haushaltsentscheidungsprozesse in den kreisfreien Städten der Bundesrepublik », dans Dieter Schimanke (Hrsg.), Stadtdirektor oder Bürgermeister. Beiträge zu einer aktuellen Kontroverse, Bâle, Boston, Stuttgart, Birkhäuser Verlag, 1989 (Stadtforschung Aktuell, Bd. 23). 
ment dans les politiques de "gestion de la crise», économique et écologique ${ }^{1}$ ?

Enfin, en Allemagne comme dans d'autres pays industrialisés, l'intervention croissante des collectivités locales dans les politiques dites de développement économique fait l'objet d'une part importante des analyses de politiques publiques; celles-ci prennent un relief particulier depuis l'intégration des cinq nouveaux Länder de l'Est, qui se traduit par une subite accentuation des disparités spatiales de développement économique, auparavant présentées globalement et sans doute grossièrement en termes d'opposition entre les Länder du Nord et ceux du Sud ${ }^{2}$. Ces travaux, qui s'inspirent fréquemment des théories du post-fordisme et de la régulation, s'intéressent en particulier aux mécanismes de mobilisation par les collectivités territoriales des potentialités et ressources endogènes, et de coordination de systèmes territoriaux à plusieurs acteurs, élargissant considérablement la conception traditionnelle que l'on avait de la Kommunalpolitik $^{3}$. Etudiant cette Politik der dezentralen Eigenentwicklung,

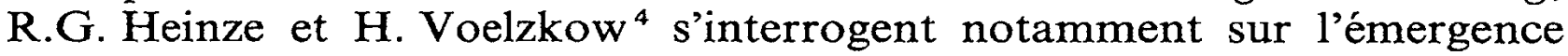
de structures de «corporatisme local et régional ».

\section{FRANCE : POLITIQUE ET DÉCENTRALISATION}

La première décennie de la décentralisation (lois de 1982-1983) a donné lieu à une importante production de recherche en France : l'analyse en termes de gouvernement local se développe surtout dans des analyses d'espace politique et de nouveaux modes d'action publique ${ }^{s}$.

1. H.E. Maier, H. Wollmann (Hrsg.), Lokale Beschäftigungspolitik, Boston, Stuttgart, Birkhäuser Verlag, 1986 (Stadtforschung Aktuell, Bd. 10); B. Blanke, E. Adalbert, H. Wollmann (Hrsg.), Die Zweite Stadt. Neue Formen lokaler Arbeits- und Sozialpolitik, Opladen, Westdeutscher Verlag, 1986 (Leviathan, Sonderheft 7) ; B. Blanke, H. Heinelt, C.W. Macke, Großstadt und Arbeitslosigkeit. Ein Problemsyndrom im Netz lokaler Sozialpolitik, Opladen, Westdeutscher Verlag, 1987 (Studien zur Sozialwissenschaft, Bd. 73); W. Jaedieke et al., Lokale Politik..., op. cit.; A. Evers, H.G. Lange, H. Wollmann (Hrsg.), Kommunale Wohnungspolitik, Stuttgart, Birkhäuser Verlag, 1983 ; J. Hucke, R. Ueberhorst (Hrsg), Kommunale Umweltpolitik, Bâle, Boston, Stuttgart, Birkhäuser Verlag, 1983 (Stadtforschung Aktuell, Bd. 5).

2. R.G. Heinze, H. Voelzkow, "Regionalisierung der Strukturpolitik in NordrheinWestfalen », dans Bernhard Blanke (Hrsg.), Staat und Stadt. Systematische, vergleichende und problemorientierte Analysen "dezentraler Politik», Opladen, Westdeutscher Verlag, 1991.

3. M. Mayer, "Postfordismus" and "lokaler Staat" ", dans Hubert Heinelt, Hellmut Wollmann (Hrsg.), Brennpunkt Stadt. Stadtpolitik und lokale Politikforschung in den 80er und 90er Jahren, Bâle, Boston, Stuttgart, Birkhäuser Verlag, 1990 (Stadtforschung Aktuell, Bd. 31) ; M. Mayer, "Neue Trends in der Stadtpolitik - eine Herausforderung für die Lokale Politikforschung", dans Bernhard Blanke (Hrsg.), Staat und Stadt. Systematische, vergleichende..., op. cit.

4. R.G. Heinze, H. Voelzkow, "Kommunalpolitik und Verbände : Inszenierter Korporatismus auf lokaler und regionaler Ebene? ", dans Hubert Heinelt, Hellmut Wollmann (Hrsg.), Brennpunkt Stadt. Stadtpolitik und lokale Politikforschung in den 80er und 90er Jahren, Bâle, Boston, Stuttgart, Birkhäuser Verlag, 1991 (Stadtforschung Aktuell, Bd. 31). p. 3.

5. J.-L. Briquet, F. Sawicki, "L'espace du local », Politix, 7-8, octobre-décembre 1989, 


\section{Richard Balme et al.}

Il existait déjà en France une tradition d'analyse d'un niveau politique local différencié ${ }^{1}$ : les tenants de l'approche communautaire, qui remettaient en cause l'idée d'une communauté locale unie et apolitique ${ }^{2}$; les travaux du CSO (Centre de sociologie des organisations) sur l'interdépendance des acteurs centraux et périphériques ${ }^{3}$; enfin, des auteurs de la sociologie marxiste analysant les rapports de pouvoir localisés en milieu urbain ${ }^{4}$. Ces travaux allaient déjà à l'encontre d'une approche institutionnelle et juridique, longtemps hégémonique, réduisant le politique au niveau du seul Etat-nation, et selon laquelle les collectivités locales étaient exclusivement confinées dans des actions administratives, vision d'ailleurs confortée par l'historique de la politique française d'aménagement $\mathrm{du}$ territoire ${ }^{s}$.

Les problématiques des recherches récentes ne peuvent s'appréhender sans le rappel d'un double contexte. D'une part, celui de la décentralisation, de la répartition des compétences entre les différents niveaux locaux, sans pour autant que les inégalités spatiales aient disparu, au détriment surtout de vastes espaces ruraux. Celui de l'aménagement du territoire, d'autre part, qui se défait et laisse la place à un pilotage économique localisé. En effet, les dynamiques économiques inégales des territoires $^{6}$, le renforcement du tropisme de l'intégration européenne ${ }^{7}$ et le dessaisissement croissant des interventions économiques de l'Etat au profit des collectivités territoriales renversent le statut et les pratiques de l'aménagement du territoire. Celui-ci ne disparaît pas véritablement de l'agenda politique mais s'y réinscrit de façon rénovée, plus localisée.

Dans ce contexte, les recherches de la science politique française consacrées à cette question sont elles-mêmes l'objet de mutations, tant par la quête de nouveaux paradigmes que par les rapprochements avec d'autres disciplines (économie, aménagement ou géographie). Elles se caractérisent souvent par une approche en termes d'évaluation, par un retour à l'empirisme, à la typologie et à l'observation du terrain. Ces travaux se révèlent largement en quête de nouveaux modes opératoires

1. A. Mabileau, "Les institutions locales et les relations centre-périphérie », dans M. Grawitz, J. Leca (dir.), Traité de science politique, tome 2, Paris, PUF, 1985; Y. Mény, "Les politiques des autorités locales", dans M. Grawitz, J. Leca (dir.), Traité de science politique, tome 4, Paris, PUF, 1985.

2. L. Wylie, Chanzeaux, village d'Anjou, Paris, Gallimard, 1970.

3. J.-P. Worms, "Le préfet et ses notables», Sociologie du travail, 3, 1966 ; M. Crozier, J.-C. Thoenig, «La régulation des systèmes organisés complexes. Le cas du système de décision politico-administratif local en France ", Revue française de sociologie, 16,1975 , p. 3-32; P. Grémion, Le pouvoir périphérique. Bureaucrates et notables dans le système politique français, Paris, Le Seuil, 1976.

4. M. Castells, F. Godard, Monopolville. Analyse des rapports entre l'entreprise, l'Etat et l'urbain à partir d'une enquête sur la croissance industrielle et urbaine de la région de Dunkerque, Paris, La Haye, Mouton, 1974.

5. J.-P. Laborie, J.-F. Langumier, P. De Roo, La politique d'aménagement du territoire français, Paris, La Documentation française, 1985; C. Lacour, P. Delfaud, J. Lajugie, Espace régional et aménagement du territoire, Paris, Dalloz, 1985.

6. X. Greffe, Territoires en mutation, Paris, Economica, 1984; R. Uhrich, La France inverse? Les régions en mutation, Paris, Economica, 1987.

7. P. Muller, «Entre le local et l'Europe. La crise du modèle français de politiques publiques $»$, Revue française de science politique, 42, (2), avril 1992, p. 275-297. 


\section{Les politiques territoriales en Europe de l'Ouest}

pour les politiques publiques ${ }^{1}$. Les recherches qui rendent compte de ces phénomènes s'inscrivent souvent dans des lectures juridiques, institutionnelles ou décisionnelles. Celles qui dépassent ces postures multiplient les interrogations sur l'efficience des stratégies des acteurs locaux et sur la structure organisationnelle optimale pour le gouvernement local. In fine apparaît le constat du maintien d'une régulation étatique et de sa nécessité pour certains acteurs ou espaces défavorisés.

\section{LOGIQUE DE LA DÉCENTRALISATION}

Rien ne serait plus inexact que de dater le démarrage de la logique décentralisatrice du début des années 1980. Dans son histoire politique et administrative de la décentralisation, V.A. Schmidt utilise cette formule : "When informal rules become formal roles $»^{2}$.

Vers la fin des années 1970, des chercheurs du CERAT (Centre de recherche sur le politique, l'administration et le territoire, Grenoble) avaient déjà souligné l'existence d'un modèle d'organisation territorial dans lequel le centre et les élus locaux étaient mis en relation, et les demandes locales placées en position centrale ${ }^{3}$. L'Etat l'actualisait en alternance avec un modèle sectoriel, selon les phases de changement et de stabilisation. Au début des années 1980, les lois de décentralisation légitiment et institutionnalisent le système local: celle du 2 mars 1982 sur «les droits et libertés des communes, des départements et des régions »; de janvier et juillet 1983 sur les compétences locales; de juillet 1985 sur l'urbanisme; de 1984 et 1987 sur le statut de la fonction publique territoriale ${ }^{4}$. La loi de 1982 accélère le principe de l'intervention économique des collectivités locales, particulièrement en faveur de la région, associée à l'élaboration du plan national (loi du 29 juillet 1982). $\mathrm{Au}$ total, les observateurs s'accordent à reconnaître à la commune le niveau de distributeur des services, au département l'organisation de la solidarité locale, et à la région l'aménagement du territoire et le développement économique et social. Ce consensus se retrouve aussi pour constater le développement des interventions économiques des collectivités locales ${ }^{5}$ et les pratiques entrepreneuriales des maires urbains ${ }^{6}$.

1. S. Wachter (dir.), Politiques publiques et territoires, Paris, L'Harmattan, 1989 ; $\mathrm{S}$. Wachter, «Ajustements et recentrage d'une politique publique : le cas de l'aménagement du territoire ", Sociologie du travail, 1, 1989, p. 51-74.

2. V.A. Schmidt, Democratizing France: the political and administrative history of decentralization, Cambridge, Cambridge University Press, 1991.

3. F. d'Arcy, Territoires en gestation, Paris, DGRST, 1979.

4. G. Gontcharoff, S. Milano, La décentralisation, nouveaux pouvoirs, nouveaux enjeux, Paris, Syros, 1983.

5. F. Gerbaux, P. Muller, «Les interventions économiques locales », Pouvoirs, 60, $1992,99-114$.

6. A. Faure, "Pouvoir local en France: le management mayoral à l'assaut du clientélisme », Politiques et management public, 9 (3), septembre 1991, p. 115-132; J.G. Padioleau, "L'action publique urbaine moderniste", Politiques et management public, 9 (3), septembre 1991, p. 133-146. 


\section{Richard Balme et al.}

En dernier lieu, la loi d'orientation relative à l'administration territoriale de la République, adoptée le 24 janvier 1992, s'inscrit dans cet ajustement de l'Etat aux gestions localisées. Des dispositifs sont destinés à la coopération entre collectivités locales - communauté de communes et de villes, ententes interrégionales et coopération transfrontalière - en vue surtout de l'aménagement de l'espace et du développement économique. La priorité est également donnée à la déconcentration qui structure de plus en plus la logique décentralisatrice, et au renforcement du rôle de l'Etat au niveau régional, pour en adapter l'organisation territoriale. Ce renouvellement de l'autorité étatique, à travers les préfets, par exemple, comme "chefs d'orchestre " ${ }^{1}$, affirme sa vocation à mieux coordonner sur le terrain les transversalités interministérielles.

La décentralisation comme "processus incrémental de réforme ${ }^{2}{ }^{2}$ soulève les limites de cette capacité d'action des gouvernements locaux ${ }^{3}$. La «nouvelle rhétorique du bon management ${ }^{4}$ implique tout d'abord un rôle fondamental de la communication dans la mise en valeur et en réseaux des territoires ${ }^{5}$. Cet impératif de la «symbolique du changement ${ }^{6}{ }^{6}$, s'il a des effets pratiques en termes de stratégies de pouvoir et de légitimation, ne saurait dissimuler les inégales capacités des acteurs locaux à conduire des politiques publiques. De nombreux auteurs s'accordent ainsi à reconnaître l'apparition de tutelles entre élus ${ }^{7}$ et entre collectivités locales, de la ville-centre, par exemple, sur les petites communes voisines ou du département sur des communes rurales ${ }^{8}$. Ils soulignent également le travers de la concurrence entre collectivités locales ${ }^{9}$ et le risque de l'aménagement d'un chacun pour soi ${ }^{10}$, avec le « technopôle comme expression d'un aménagement du territoire concurrentiel ${ }^{11}$. Dans la recherche de la réduction des disparités spatiales, particulièrement face à la mise en place d'un nouvel espace public européen et de sa norme de subsidiarité, la confirmation de territoires en perdition fait émerger le besoin d'une régulation étatique ${ }^{12}$. Et le contrôle technique des services d'urbanisme, les procédures contractuelles

1. C. Grémion, «Que reste-t-il des administrations déconcentrées?», dans P. Muller (dir.), L'administration française est-elle en crise?, Paris L'Harmattan, 1992.

2. J.-C. Thoenig, «La décentralisation, dix ans après », Pouvoirs, 60, 1992, p. 5-16.

3. P. Muller (dir.), L'administration française est-elle en crise?, op. cit.

4. A.V. Schmidt, Democratizing France..., op. cit. 1990.

5. H. Bakis (dir.), Communications et territoires, Paris, La Documentation française,

6. J.-L. Marie, "La symbolique du changement », dans A. Mabileau, C. Sorbets (dir.), Gouverner les villes moyennes, Paris, Pedone, 1989.

7. J. Rondin, Le sacre des notables. La France en décentralisation, Paris, Fayard, 1985 ; Y. Mény, "La république des fiefs », Pouvoirs, 60, 1992, p. 17-24.

8. J.-C. Thoenig, «Le grand horloger et les effets de système: de la décentralisation en France », Politiques et management public, 1, mars 1985, p. 135-158.

9. M. Crozier, "Partage des pouvoirs : la décentralisation restera inachevée tant que ce partage ne sera pas réel $»$, Le Quotidien du maire, 13-19 mai, 1992, p. 8.

10. P. Téqueneau, "Les territoires après la décentralisation: le dessous des cartes", Hérodote, 62, 1991, p. 44-63.

11. J. Dumas, «Le technopôle comme expression d'un aménagement du territoire concurrentiel : l'Etat, la ville, la région", intervention au colloque "Nouvelles industrialisations, nouvelle urbanité, villes et technopoles », Toulouse, septembre 1987.

12. J.-P. Balligand, D. Maquart, La fin du territoire jacobin, Paris, Albin Michel, 1990. 
Etat-région ou les mesures de péréquation financière adoptées en 1991 pour corriger les disparités entre collectivités locales sont interprétés par certains comme les signes d'une recentralisation ${ }^{1}$.

\section{Développement local et aménagement du territoire}

Le contexte spatio-économique n'en demeure pas moins très inégalitaire $^{2}$. Les paradigmes de la géographie et de l'économie soulignent le changement de déterminisme des territoires dans le cadre européen, par exemple sur un axe Nord-Sud, et la polarisation du développement autour de l'axe central européen ${ }^{3}$. Un paradigme plus politiste insiste tout autant sur la modernisation du gouvernement des grandes villes dans la gestion des problèmes urbains et des rapports à l'Etat central ${ }^{4}$, et sur l'affirmation des villes moyennes ${ }^{5}$. Par ailleurs, nous ne sommes plus en présence de l'aménagement du territoire volontariste et centralisateur des années $1960^{\circ}$. Les analyses s'accordent à expliquer cet abandon par l'inadaptation et la faible efficacité des outils d'intervention classiques, les effets de la crise économique, le transfert des compétences économiques vers les collectivités locales et le renforcement des effets de l'intégration européenne.

A contrario, dans un contexte de remise en cause des politiques distributives de l'Etat-providence, l'idée de développement local se révèle un paradigme largement consensuel dans les discours et pratiques des politiques locales, tout comme chez les analystes du phénomène ${ }^{7}$. Sa dynamique est essentiellement endogène et réticulaire ${ }^{8}$. Les principaux facteurs d'une politique locale de développement sont souvent une relative autonomie de décision, des ressources propres, une composition sociale favorable de sa population, un certain consensus sur la politique locale, voire une situation historique d'opposition au pouvoir central ${ }^{9}$. On peut d'ailleurs référer la diffusion de ce paradigme aux déplacements qui s'opèrent chez les spécialistes qui en rendent compte : il s'agit essentiellement des économistes, des aménageurs et des géographes, souvent dans un cadre d'expertise.

1. J.-C. Douence, L'action économique locale, décentralisation

Paris, Economica, 1988.

2. B. Planque (dir.), Le développement décentralisé. Dynamique spatiale de l'économie et planification régionale, Paris, Litec, 1983 (coll. «GRAL »).

3. C. Lacour et al., Espace régional..., op. cit.; R. Brunet (dir.), Les villes européennes, Paris, La Documentation française, 1989; J. de Gaudemar, R. Prud'homme, «Spatial impacts of deindustrialization in France ", dans L. Rodwin, H. Sazanami (eds), Industrial change and regional economic transformation. The experience of Western Europe, Londres, Harper Collins, 1991, p. 105-136.

4. D. Lorrain, "De l'action républicaine au gouvernement urbain», Sociologie du travail, 4, 1991, p. 461-484.

5. A. Mabileau, C. Sorbets (dir.), Gouverner les villes moyennes, op. cit.

6. J. Palard, «L'aménagement du territoire à l'épreuve de la décentralisation et de l'intégration européenne », Annuaire des collectivités locales, 1993 (à paraître).

7. B. Pecqueur, Le développement local, Paris, Syros/Alternatives, 1989.

8. C. Neuschwander, "Territoire, démocratie. Les conditions de l'efficacité », Territoires, janvier-février 1990, p. 19-26; G. Benko, A. Lipietz, Les régions qui gagnent, Paris, PUF, 1992.

9. O. Benoit-Guilbot, «Les acteurs locaux du développement économique local : y at-il un "effet localité" ?", Sociologie du travail, 4, 1991, p. 453-459. 
Richard Balme et al.

Au total, avec ce dialogue loin d'être stabilisé entre l'Etat et les collectivités locales, la prolifération des systèmes locaux pose aux chercheurs la difficulté d'une théorisation de la généralité territoriale. Cependant, une grille de lecture par effets induits, repérant les événements qui changent et structurent de façon significative les modes de régulation du système, permet de mieux apprécier la décentralisation ${ }^{1}$. En s'éloignant d'une trame causale de la décision d'une politique publique, il s'agit plutôt de relever les interférences, les effets pervers et les dysfonctions, leur agrégation fournissant autant d'indicateurs, indirects mais décisifs, de la décentralisation.

Bien que transitoire, la nature des relations entre l'Etat et les collectivités locales offre ainsi le constat d'un jeu à somme positive entre les acteurs, dans un cadre de plus en plus contraint par la construction européenne $^{2}$. La décentralisation n'a pas entièrement dessaisi les fonctions de l'Etat au profit des seules collectivités locales. Nous sommes plutôt en présence d'une interdépendance élargie. De fait, les recherches s'interrogent moins sur la hiérarchie fonctionnelle entre collectivités locales que sur les transversalités, les modes de coopération et les actions en réseaux. Cette prégnance du paradigme inter-organisationnel permet tout autant de relativiser l'aspect idéologique des discours sur le développement local que l'éventualité d'un "fondamentalisme» du local exclusivement considéré dans son autonomie ou son autarcie ${ }^{3}$.

\section{ITALIE : FRAGMENTATION ET NÉOLOCALISME}

En Italie, toute question territoriale est placée sous le signe d'une double contradiction. La première, fondatrice, est celle d'une intégration nationale inaboutie : toutes les politiques territoriales sont perturbées par ce problème et, en conséquence, soumises aux aléas des enjeux politiques qui lui sont liés. La seconde tient à la relative anarchie politico-institutionnelle ainsi créée : les politiques territoriales peuvent y être aussi bien des échecs retentissants (la politique de développement du Mezzogionno, par exemple) que des réussites pragmatiques (la politique des régions du centre, par exemple). La recherche italienne s'est donc efforcée d'analyser ces contradictions et a presque toujours été conduite, pour ce faire, à dépasser les analyses en termes d'acteurs et d'institutions pour privilégier les processus socio-politiques qui les sous-tendent, qu'elle les aborde sous l'angle historique, politique ou sociologique.

1. J.-C. Thoenig, "Le grand horloger... », art. cité.

2. R. Balme, A. Mabileau, "Les collectivités locales, l'Etat, et l'Europe », dans L'état du monde, Paris, La Découverte, 1992, p. 493-496.

3. G. Benko, A. Lipietz, Les régions qui gagnent, op. cit. 
CENTRE ET PÉRIPHÉRIES :

L'HISTOIRE D'UNE DIFFICILE CONSTRUCTION NATIONALE

Comme l'écrit ironiquement S. Cassese : « Si on approfondit la question en termes scientifiques, on se rend compte qu'une périphérie peut être centrale... Le centre, en outre, peut être polycentrique... Mais l'autre terme n'est pas non plus univoque, car il existe non pas une périphérie, mais plusieurs. Et les rapports entre elles ne sont pas moins importants que les rapports entre centre et périphérie. Bref, tous les chemins ne mènent pas à Rome ${ }^{1}$. L'explication de la complexité de ces rapports en Italie est à chercher dans l'histoire d'un Etat-nation et de son territoire qu'on a pu qualifier de polycentriques ou de fragmentés ${ }^{2}$. Sans cette dimension historique, il n'est pas possible de comprendre les aléas d'une régionalisation inscrite dans la Constitution de 1948, mais seulement partiellement appliquée dans les années suivantes avec l'instauration de cinq régions à statut spécial, pour être ensuite politiquement bloquée durant vingt-cinq ans, jusqu'aux lois de 1970-1972 qui instituent les quinze régions à statut ordinaire ${ }^{3}$. Il n'est pas non plus possible d'interpréter une politique d'aménagement du territoire velléitaire dans les meilleurs des cas, totalement pervertie dans le cas de la tentative de développement du Mezzogiorno ${ }^{4}$.

La généalogie de l'idée régionale en Italie est particulièrement révélatrice. Elle démontre en effet que l'idéal régionaliste, voire fédéral, imprégnait les pères de l'unité italienne, et que seule la profonde instabilité d'un pays artificiellement unifié les poussa à adopter les thèses centralistes napoléoniennes. Le fascisme renforça cette tendance, inspirant par là même à ses opposants des aspirations régionalistes. C'est cette inspiration que reprend la Constitution de 1948, par souci d'anti-autoritarisme et par nécessité d'affronter une question méridionale de plus en plus pressante ${ }^{5}$. Le blocage politique de la régionalisation, destiné à préserver l'hégémonie démocrate-chrétienne d'une prise de pouvoir de la gauche par la périphérie, a donc encore accentué le quiproquo entre l'Italie et son histoire. Son application a été à la fois une tentative de dépasser la grave crise politique des années 1970, mais aussi « rien moins qu'une étape de l'unification nationale, l'affirmation du pluralisme institutionnel, un moyen d'intégrer des courants centrifuges $"{ }^{6}$. Cette récon-

1. S. Cassese, "Centro e periferia in Italia. I grandi tornanti della lora storia », Rivista trimestrale di diritto pubblico, 36, 1986.

2. S. Tarrow, Between center and periphery. Grassroots politicians in Italy and France, Londres, Yale University Press, 1977 ; S. Tarrow, "Decentramento incompiuto o centralismo ristorato? L'esperienza regionalistica in Italia $\mathrm{e}$ in Francia », Rivista italiana di scienza politica, 9, 1979; B. Dente, Governare la frammentazione. Stato, regioni e ente locali in Italia, Bologne, Il Mulino, 1985.

3. ISAP, La regionalizzazione, 2 vol., Milan, Giuffrè, 1983 ; D. Mazzega, J. Musitelli, L'organisation régionale en Italie, Paris, La Documentation française, 1980 (Notes et études documentaires 4553-4554).

4. P. Allum, "Thirty years of Southern policy in Italy », Political Quarterly, 52, 1981.

5. L. Gambi, "Le "regioni" italiane come problema storico", Quaderni storici, 34, 1977 ; P. Bonora, Regionalità. Il concetto di regione nell'Italia del secondo dopoguerra (19431970), Milan, Angeli, 1984.

6. D. Mazzega, J. Musitelli, L'organisation régionale..., op. cit. 


\section{Richard Balme et al.}

ciliation avec l'histoire s'est pourtant vite révélée être un chemin difficile : les institutions étatiques tentent sans cesse de limiter et de contrôler les pouvoirs dévolus aux régions; les réseaux politiques, comme les intérêts organisés, traditionnellement structurés autour de la commune et de la province, tardent à s'investir à l'échelon régional. Décentralisation incomplète, régionalisation paradoxale, dit-on ${ }^{1}$. Le surgissement du mouvement des Ligues régionales dans le Nord du pays, mouvement protestataire et de prétentions fédéralistes, atteste bien du malaise qui mine encore aujourd'hui l'intégration nationale italienne: les périphéries riches protestent non pas tant au nom d'une appartenance culturelle, comme le ferait un mouvement régionaliste classique, mais au nom du rejet du centre et des périphéries pauvres. A la faveur de la crise politique, elles retournent ainsi à leur profit le localisme traditionnel de la vie politique italienne pour lui donner la figure originale d'un «populisme régionaliste $\gg^{2}$.

\section{POLITIQUE DU PRAGMATISME INSTITUTIONNEL}

Déjà les travaux de $\mathrm{S}$. Tarrow ${ }^{3}$ montraient que l'application du modèle politico-administratif jacobin s'était heurtée à la puissance de réseaux locaux alimentés par des appartenances municipales, communautaires ou clientélistes : la faiblesse de l'organisation centrale a laissé le champ libre à l'organisation de réseaux locaux cherchant à s'accaparer les ressources publiques. Entre centre et périphéries, l'élu est tenu de se comporter comme un «entrepreneur politique», tentant de maximiser ses ressources en exploitant la multiplicité des sites de pouvoir. C'est pourquoi les partis politiques ont pu longtemps être considérés comme assurant la centralité politique : par l'intermédiaire des réseaux partisans s'effectue un échange politique vertical par lequel les périphéries parviennent à contrôler le centre. L'actuelle crise des partis politiques italiens est ainsi un signe majeur de la crise du système politique tout entier: avec eux s'écroule tant le seul processus politique d'intégration nationale que le vecteur essentiel de l'échange politique ${ }^{4}$.

Or des études récentes attestent que l'échange politique intensif est la clé aussi bien des réussites du système que de ses échecs. C'est ainsi que l'échange clientéliste (colonisation de la bureaucratie par un parti, politique de distribution clientéliste des ressources, détournement des facultés de mobilisation collective) est à l'origine de l'enlisement des politiques publiques et des actions politiques dans le Mezzogiorno ${ }^{5}$ : on

1. S. Tarrow, "Decentramento... », art. cité ; C. Trigilia, « Il paradosso della regione : regolazione economica e rappresentanza degli interessi », Meridiana, 6, 1989.

2. R. Biorcio, dans R. Mannheimer (ed.), La Lega lombarda, Milan, Feltrinelli, 1991.

3. S. Tarrow, Between..., op. cit. ; «Decentramento...», art. cité.

4. A. Pizzorno, "Scambio politico e identità colletiva di classe », Rivista italiana di scienza politica, 7 (2), 1977 ; F. Cazzola, "L'associazionismo istituzionalizzato », dans M. Fedele (ed.), Il sistema politico locale, Bari, De Donato, 1983.

5. L. Graziano, Clientelismo e sistema politico, Milan, Angeli, 1980; M. Caciagli, Democrazia cristiana e potere nel Mezzogiorno, Florence, Guaraldi, 1975; S. Cafiero, Tradizione e attualità del meridionalismo, Bologne, Il Mulino, 1989. 
sait qu'on va aujourd'hui jusqu'à mettre en doute la capacité même de l'appareil d'Etat à affronter le problème des inégalités de développement. A contrario, B. Dente ${ }^{1}$ montre, en étudiant les échanges entre niveaux de gouvernement, que la fragmentation politique italienne a, en quelque sorte, anticipé sur la complexité contemporaine des relations intergouvernementales, et qu'elle peut être source de performances institutionnelles si elle est acceptée et maîtrisée. La longue observation des régions à statut ordinaire par une équipe italo-américaine a encore approfondi la connaissance de ce pragmatisme italien ${ }^{2}$. Menée en termes d'écologie du rendement institutionnel, ces études effectuent un classement des régions selon leurs performances institutionnelles, des plus performantes (Emilie-Romagne, Lombardie, Ombrie, Toscane, Vénétie) aux moins efficaces (Calabre, Molise, Campanie). Le degré de performance est fortement corrélé au niveau de développement économique pour les régions peu développées et peu performantes. Un profond changement démographique peut expliquer les contre-performances de certaines régions riches (Piémont, Ligurie, Latium). Mais c'est au total une culture politique fortement mobilisatrice qui y apparaît comme le déterminant du succès institutionnel : « La participation civique explique le développement socio-économique, et non l'inverse. La culture politique, et non la structure socio-économique, est le déterminant principal $\mathrm{du}$ rendement institutionnel $\gg{ }^{3}$. Ces analyses politico-institutionnelles rejoignent ainsi l'important courant de sociologie des territoires, qui s'est développé depuis quelques années en Italie.

\section{DES TERRITOIRES SOCIO-POLITIQUES DIFFÉRENCIÉS}

Des recherches de sociologie économique désormais classiques ont établi l'existence de «trois Italie», trois formations sociales différenciées $^{4}$. Ces trois formations sociales territorialisées ne connaissent ni le même mode de développement économique, ni le même type de régulation sociale et politique. Celle du Nord, organisée autour des grandes métropoles, correspond au modèle qui associe industrialisation classique, urbanisation, différenciation sociale et statuts acquis, mobilisation sectorielle, vote d'opinion. Celle du Mezzogiorno (le Sud de Rome et les îles) a pour caractéristiques une économie marginale et assistée, une régulation sociale organisée autour de la cellule familiale, une anomie de la mobilisation collective, un échange politique à règle clientéliste et un vote d'obédience. A cette dichotomie classique, héritée du méridio-

1. B. Dente, Governare..., op. cit.

2. R.D. Putnam, R. Leonardi, R.Y. Nanetti, La pianta e le radici. Il radicamento dell'istituto regionale nel sistema politico italiano, Bologne, Il Mulino, 1985 ; R.Y. Nanetti, Growth and territorial policies. The Italian model of social capitalism, Londres, Pinter, 1988; R.D. Putnam, «Rendimento istituzionale e cultura politica », Polis, 3, 1988.

3. R.D. Putnan, « Rendimento... », art. cité.

4. A. Bagnasco, Tre Italie. La problematica territoriale dello sviluppo italiano, Bologne, Il Mulino, 1977 ; G. Fuà, C. Zacchia (eds), Industrializzazione senza fratture, Bologne, II Mulino, 1983. 


\section{Richard Balme et al.}

nalisme, s'est ajoutée la détermination de la "troisième Italie » des régions du centre et du Nord-Est. Celle-ci est caractérisée par des réseaux performants de petites entreprises, un réseau urbain très dense, une régulation sociale réticulaire et participative, une mobilisation associative forte et un vote d'identité (stabilité des cultures politiques locales): l'originalité de cette formation sociale territorialisée a focalisé de nombreux travaux ${ }^{1}$.

C'est dans ce cadre théorique que se situe désormais l'approche du mal-développement méridional ${ }^{2}$. Le débat sur la question méridionale s'est transformé ces dernières années : certes, toujours animé par une approche économique du mal-développement et de l'échec des politiques publiques, il est de plus en plus sensible aux dimensions sociales et politiques du syndrome méridional. L'observation de voies de développement diverses dans les sociétés méridionales (en particulier la relative réussite de régions comme les Abbruzes et la Molise dans le secteur des PME) a imposé l'idée que le Mezzogiorno n'est pas une société homogène. Face à ces destins différents, la recherche en est venue de plus en plus à interroger les caractères socio-politiques de ces sociétés, complexifiant ainsi l'idée classique de sociétés dépendantes. Pourquoi ces sociétés sont-elles déficitaires en mobilisation collective ? Pourquoi la médiation politique est-elle toujours captée par une «classe de régime» qui monopolise l'accès aux ressources publiques? Comment le passage du patronage classique au clientélisme de masse a-t-il aggravé la situation ${ }^{3}$ ? On le sait, ces questions sont, en Italie, au cœur même de la crise de la norme étatique, d'un Etat qui n'est pas parvenu à assurer l'intégration nationale, d'un appareil d'Etat parasité par les clientélismes ${ }^{4}$.

Cette différenciation territoriale en termes socio-économiques est encore complexifiée par l'existence de subcultures politiques territorialisées, particulièrement nettes dans la «troisième Italie » ${ }^{5}$. Les travaux qui leur sont consacrés y démontrent une grande continuité de l'orientation politique depuis l'Unité, des mécanismes d'association et de mobilisation très denses, une socialisation politique permanente par la famille et les groupes d'appartenance : les uns et les autres sont de coloration « rouge»

1. A. Bagnasco, La costruzione sociale del mercato, Bologne, Il Mulino, 1988 ; A. Bagnasco, C. Trigilia, Società e politica nell'aree di piccola impresa. Il caso di Bassano, Venise, Arsenale Editrice, 1984; A. Bagnasco, C. Trigilia, Società e politica nell'aree di piccola impresa. Il caso della Valdelsa, Milan, Angeli, 1985; E. Ritaine, «La modernité localisée? Leçons italiennes sur le développement régional ", Revue française de science politique, 39 (2), avril 1989.

2. C. Trigilia, "Le condizioni " non economiche" dello sviluppo: problemi di ricerca sul Mezzogiorno d'oggi », Meridiana, 1 et 2, $1988 ;$ E. Wolleb, G. Wolleb, Divari regionali $e$ dualismo economico, Bologne, Il Mulino, 1990.

3. Sur l'ensemble de ce débat, voir F.P. Cesare (éd.), Dopo il familismo cosa? Tesi a confronto sulla questione meridionale negli anni' 90, Milan, Angeli, 1992; R. Catanzaro, "Struttura sociale, sistema politico e azione colletiva nel Mezzogiorno », Stato e Mercato, $8,1983$.

4. S. Pallida, "L'anamorphose de l'Etat-nation. Le cas italien», Cahiers internationaux de sociologie, 93, 1992; R. Cartocci, "Localismo e protesta politica », Rivista italiana di scienza politica, 3, 1991.

5. C. Trigilia, «Le subculture politiche territoriale», Quaderni Feltrinelli, 16, 1981 ; C. Trigilia, Grandi partiti e piccole imprese. Comunisti e democristiani nelle regioni a economia diffusa, Bologne, Il Mulino, 1986. 
(socialo-communiste) en Italie centrale, et de coloration "blanche» (démocrate-chrétienne) en Italie nord-orientale ${ }^{1}$. Certes, ces contextes politiques homogènes sont perturbés par l'actuelle désaffection vis-à-vis du politique; aucune étude en profondeur ne permet pour l'instant de prévoir si ces ressources socio-politiques vont se réinvestir différemment. Il apparaît pourtant d'ores et déjà évident qu'elles constituent des facteurs de résistance différents à l'implantation des Ligues : celles-ci progressent plus aisément dans les contextes de culture démocrate-chrétienne, alors qu'un contexte traditionnel de culture «rouge», doté d'une plus grande vigilance démocratique, bloque leurs avancées ${ }^{2}$.

La recherche italienne s'est orientée ces dernières années vers un concept de moyenne portée, qui permet d'analyser plus précisément les processus de médiation politique : la capacité à organiser et à mobiliser les représentations des intérêts locaux. Une culture politique forte semble, en effet, doter les acteurs politiques locaux ou régionaux de cette capacité qui permet souvent de surmonter les faiblesses institutionnelles. Cette capacité est au principe, par exemple, des bonnes performances de la région Emilie-Romagne, capable, dans un contexte politique très homogène, de coopérer avec la plupart des acteurs collectifs ${ }^{3}$. Mais c'est aussi cette capacité qui fait encore défaut à bien des régions italiennes pour être performantes ${ }^{4}$. En effet, le "néolocalisme", cette forme de régulation locale entre marché, structures sociales et structures politiques, que les Italiens ont conceptualisée en parallèle au néocorporatisme, trouve encore pour $1^{\prime}$ heure ses plus belles illustrations au niveau local ${ }^{5}$, et ne parvient pas à s'actualiser au niveau régional, pourtant plus adaptatif face aux enjeux européens.

\section{ESPAGNE : «L'ÉTAT DES AUTONOMIES »}

En Espagne, depuis la transition démocratique qui a suivi la mort du général Franco en 1975 , les relations entre le centre et la périphérie se sont transformées de manière rapide et profonde, comme sans doute dans aucun des autres pays de l'Europe occidentale, faisant de ce pays un cas largement singulier. Elles ont suscité une littérature extrêmement abondante et variée non seulement en espagnol, mais également en anglais et en français, rendant impossible tout inventaire pouvant avoir

1. M. Caciagli, "Quante Italie? Persistenza e trasformazione delle culture politiche subnazionale », Polis, 3, 1988.

2. P. Natale, dans R. Mannheimer (éd.), La Lega lombarda, op. cit.

3. R. Leonardi, R.Y. Nanetti (ed.), The regions and European integration. The case of Emilia-Romagna, New York, Pinter, 1990 (Publication of the European University Institute).

4. C. Trigilia, «Il paradosso... », art. cité ; G. Dematteis, "Regioni geografiche, articolazione territoriale degli interessi e regioni istituzionali », Stato e Mercato, 27, 1989; P.C. Schmitter, L. Lanzalaco, «L'organizzazione degli interessi imprenditoriali a livello regionale ", Stato e Mercato, 22, 1988; M. Cammelli, "Regione e rappresentanza degli interessi : il caso italiano ", Stato e Mercato, 29, 1990.

5. C. Trigilia, "La regolazione localistica... », art. cité. 
Richard Balme et al.

une quelconque prétention à l'exhaustivité. De nombreuses études sont consacrées au «processus autonomique» espagnol de décentralisation ainsi qu'à la question des nationalités qui, pour une large part, est au fondement de la redéfinition des relations entre le centre et la périphérie. S'y ajoute tout un pan de travaux sur la transformation des politiques régionales, visant à réduire les fortes disparités interrégionales qui ont toujours marqué le développement de l'Espagne.

\section{LE PROCESSUS AUTONOMIQUE \\ COMME FORME SPÉCIFIQUE DE DÉCENTRALISATION}

A un modèle de relations entre le centre et la périphérie caractérisé par sa centralisation, sa simplicité, sa stabilité et l'homogénéité des structures territoriales, le processus espagnol de régionalisation a substitué brutalement, en quelques années, un tout autre modèle extrêmement décentralisé (pouvoir législatif des communautés autonomes), complexe et conflictuel, très fortement différencié dans divers domaines (compétences, statuts, territoires et même calendrier); il est aussi dynamique, car inscrit dans le cadre d'un processus de décentralisation tout à la fois «à la carte », à géométrie variable et à plusieurs vitesses ${ }^{1}$.

En ce domaine, il est clair que la mise en cuvre de la Constitution de 1978 a induit un changement de logique des relations centre-périphérie en Espagne ${ }^{2}$, à tel point que la nature elle-même de l'Etat espagnol actuel suscite des débats très nombreux, et que la complexité du processus est sans aucune commune mesure avec ce qu'il est possible d'observer en Europe occidentale, y compris en France où la politique de décentralisation engagée dans les années 1980, comparée à la régionalisation espagnole, fait figure de «changement à la marge » ${ }^{3}$.

De 1979 à 1983, dix-sept communautés autonomes ont été créées dans le cadre de différentes procédures (préférentielle, spéciale, exceptionnelle ou ordinaire, selon le cas), avec des niveaux de compétences différents se traduisant par l'élaboration d'un statut juridique spécifique à chaque communauté autonome, compétences non figées de surcroît mais susceptibles d'évoluer dans le temps, et réorganisant le territoire espagnol de manière hétérogène et non uniforme ${ }^{4}$. Au côté des dix communautés autonomes multiprovinciales, on compte ainsi sept régions constituées territorialement à partir d'une seule province. Si la Consti-

1. A. Brassloff, «Spain : the state of the autonomies », dans M. Forsyth (ed.), Federalism and nationalism, Leicester, Leicester University Press, 1989 ; "De 1'Espagne une à l'Espagne plurielle », Ramsès, 90, 1989, p. 312-327; J. Leguina Villa, Escritos sobre autonomías territoriales, Madrid, Tecnos, 1984.

2. P. Bon, F. Moderne, Les autonomies régionales dans la Constitution espagnole, Paris, Economica, 1981.

3. F. d'Arcy, M. Baena del Alcazar, Décentralisation en France et en Espagne, Paris, Economica, 1986.

4. T. Clegg, "Spain », dans E. Page, M. Goldsmith (eds), Central and local government relations, Londres, Sage, 1987 ; J. Sole-Vilanova, "Spain: developments in regional and local government », dans R. Bennet (ed.), Territory and administration in Europe, Londres, Pinter, 1989. 
tution énumère les compétences exclusives de l'Etat (au nombre de 32) et potentielles (22) des communautés autonomes ${ }^{1}$, il ne s'agit en fait que de domaines de compétences très généraux. Dans la réalité, de nombreuses compétences sont partagées et communes, bien que variables de communauté autonome à communauté autonome, occasionnant des confusions et des chevauchements permanents ${ }^{2}$, à l'origine d'un contentieux important et incessant entre l'Etat et les communautés autonomes.

Leurs relations se caractérisent par une très grande complexité à la fois juridique et politique ${ }^{3}$, et, en ce domaine, la notion $d^{2}$ « anarchies organisées » paraît s'appliquer particulièrement bien. D'autant plus que le processus autonomique affecte assez directement les relations entre communautés autonomes et provinces ${ }^{4}$. Ces relations sont elles-mêmes inégalitaires et différenciées : déficit de légitimité des Diputaciones provinciales dont les membres ne sont élus qu'au second degré parmi les conseillers municipaux, opposition entre majorités politiques différentes, différence de légitimité de l'échelon provincial selon les communautés autonomes. Ainsi la Generalitat de Catalogne ${ }^{5}$ a-t-elle tenté de faire disparaître les provinces au profit d'un nouvel échelon territorial, la comarca, alors qu'au contraire les provinces basques bénéficient d'une forte légitimité historique, liée il est vrai à des droits fiscaux particuliers ${ }^{6}$.

Le processus autonomique s'est accompagné d'une multiplication de conflits. Le Tribunal constitutionnel joue un rôle essentiel dans leur régulation juridictionnelle, mais ce mode de traitement ne parait pas de nature à combler les lacunes de la Constitution ni à résoudre des tensions qui sont essentiellement politiques ${ }^{7}$. La Constitution espagnole est souvent critiquée pour sa complexité, ses obscurités, ses ambiguïtés. L'Etat « quasi fédéral » ou " potentiellement fédéral » apparaît alors comme une sorte de monstre juridique hybride, ni unitaire ni fédéral ou, plus exactement, mi-unitaire, mi-fédéral. En ce domaine, le modèle de relations centre-périphérie en Espagne se caractérise par une originalité et une singularité certaines.

Dans ce processus autonomique spécifique à l'Espagne, il apparaît clairement que les circonstances politiques du moment de «la transition démocratique » ont pesé d'un poids déterminant. Il est alors nécessaire

1. R. Tamames, T. Clegg, «Spain: regional autonomy and the democratic transition ", dans M. Hebbert, H. Machin (eds), Regionalisation in France, Italy and Spain, Londres, LSE, 1984.

2. J.M. Castells, "Las administraciones autonómicas en la nueva fase », Revista vasca de administración pública, 22, 1989, p. 69-86; P. Subra de Bieusses, "Espagne : les autonomies dix ans après ", Pouvoirs locaux, 4, 1990, p. 116-123.

3. J. Valles, M. Cuchillo Foix, «Decentralisation in Spain : a review », European Journal of Political Research, 16 (4), 1988, p. 395-407.

4. L.M. Rebollo, "La administración local», dans E. García de Enterria (ed.), España: un presente para el futuro, vol. 2, Las instituciones, Madrid, Instituto de estudios económicos, 1984.

5. E. Martin, La Catalogne, Paris, La Documentation française, 1990.

6. E. Moxon-Browne, Political change in Spain, Londres, Routledge, 1989.

7. E. García de Enterria, "El futuro de las autonomías territoriales», dans E. García de Enterria (ed.), España: un presente para el futuro, vol. 2, Las instituciones, op. cit.; F. Moderne, "L'état des autonomies dans " 1 'Etat des autonomies" ", Revue française de droit constitutionnel, 2, 1990, p. 195-211. 


\section{Richard Balme et al.}

de voir comment l'organisation institutionnelle et territoriale de 1'Espagne est liée à la dynamique de ce qui est souvent appelé les «nationalités périphériques ».

\section{LA QUESTION DES NATIONALITÉS PÉRIPHÉRIQUES UNE PARTICULARITE ESPAGNOLE}

Le processus autonomique comme forme spécifique de décentralisation régionale et la réorganisation territoriale de l'Espagne qu'il a entraînée trouvent leur origine dans un contexte et un débat de nature essentiellement politique et idéologique, et non administrative ${ }^{1}$. Il ne s'agit pas de s'accorder sur la meilleure organisation territoriale de l'Etat en soi, mais, beaucoup plus concrètement, de trouver une réponse institutionnelle politiquement acceptable à la montée de nationalismes périphériques dans un contexte dramatique, marqué par le terrorisme et la menace de coups d'Etat militaires ${ }^{2}$. La régionalisation territoriale d'un Etat espagnol traditionnellement centralisé peut être interprétée comme résultant d'un processus de construction politique. Hormis le cas particulier basque, sur lequel on reviendra, divers auteurs s'accordent, en effet, à souligner la relative artificialité de la question «nationalerégionale » comme problème politique général et important en Espagne, et tendent à faire l'hypothèse d'une réinvention de cette question ${ }^{3}$.

En Espagne, en raison d'un contexte politique particulier, les mouvements politiques «alternatifs " remettant en cause la légitimité des Etats centraux, dans les années 1970, ont eu un écho certain dans les dernières années du régime franquiste. Dans un premier temps, une littérature spécifique est venue donner une légitimité intellectuelle à ce courant d'opinion, en réécrivant et idéalisant quelque peu l'histoire des « nationalités périphériques» espagnoles. Dans un second temps, les partis centraux de gauche (PCE, d'abord, puis PSOE et PSP) se sont saisis de cette thématique pour chercher à mobiliser les classes moyennes espagnoles qui se sont fortement développées sous le franquisme. C'est alors que la revendication d'un Etat fédéraliste et du droit à l'autodétermination des nationalités espagnoles apparait dans les programmes des partis de gauche, et que le problème de la structure territoriale de l'Espagne s'inscrit sur l'agenda politique ${ }^{4}$.

Mais ce phénomène d'appropriation thématique et cette stratégie politique des partis de gauche provoquent une surenchère des partis

1. R. Carr, "The regional problem in Spain", dans J.C. Boogman, F. Van Der Plaat (eds), Federalism, history and current significance of a form of government, La Haye, Martinus Nijhoff, 1980 ; C. Díaz López, «Center-periphery structures in Spain: from historical conflict to territorial-constitutional accomodations $"$, dans Y. Mény, V. Wright (eds), Center-periphery relations in Western Europe, Londres, Allen and Unwin, 1986.

2. E. Moxon-Browne, Political change in Spain, op. cit.

3. A. de Blas Guerrero, "El problema nacional-regional español en la transición », dans J.F. Tezanos, R. Cotarelo, A. de Blas (eds), La transición democrática española, Madrid, Sistema, 1989.

4. J.F. Tezanos, R. Cotarelo, A. de Blas (eds), La transición democrática española, op. cit.; A. de Blas Guerrero, El problema..., op. cit. 
nationalistes et autonomistes régionaux, qui doivent se démarquer des organisations politiques centrales pour continuer d'occuper régionalement leur espace politique propre. Cette radicalisation est donc très largement un effet de la concurrence entre partis politiques centraux et régionaux. En fonction, notamment, d'une identité collective régionale beaucoup plus forte mais tout à fait exceptionnelle en Espagne, cette surenchère prend au Pays Basque une forme spécifique et radicale, avec, en particulier, la stratégie politique terroriste de l'ETA qui contribue à dramatiser de façon très forte le problème national-régional et accroître son audience ${ }^{1}$. Dans ce contexte, la Constitution de 1978 constitue une réponse permettant le compromis entre la gauche socialiste et le centre, et visant à banaliser et à "noyer " d'une certaine façon la spécificité du problème basque en lui donnant un contenu plus institutionnel, élargi à l'ensemble de l'organisation territoriale de l'Etat espagnol, conditions de son acceptabilité politique. L'urgence à répondre au problème très largement spécifique du Pays Basque comme la nécessité d'habiller politiquement et juridiquement cette réponse institutionnelle conditionnent étroitement le processus autonomique et la forme singulière qu'il va prendre, tout à la fois «à la carte », à géométrie variable et à plusieurs vitesses.

Mais, dès lors que le processus est engagé, la porte est ouverte à la concurrence et à la contagion institutionnelle qui tendent, sous l'égide des partis et de leurs dirigeants régionaux, à développer une imitation et un alignement de communauté autonome à communauté autonome dans le transfert et l'exercice des compétences ${ }^{2}$. Ces phénomènes conduisent, d'une part, à la création de communautés autonomes ayant une faible identité régionale, voire aucune (Madrid, région Cantabrique, Rioja, Castille-La Manche, Castille-Léon, en particulier) et, d'autre part, à une multiplication des tensions et conflits entre l'Etat central et les communautés autonomes, qui vient nourrir le contentieux juridictionnel.

\section{LA TRANSFORMATION DE LA POLITIQUE RÉGIONALE}

De nombreux travaux se sont penchés sur les inégalités de développement entre régions, qui ont marqué l'Espagne, au début du siècle, sous le franquisme, comme encore aujourd'hui. La réponse à la question de l'évolution vers une réduction ou, au contraire, un accroissement de ces déséquilibres inter-régionaux est complexe et dépend, pour une large part, des indicateurs retenus. Mais, globalement, il ne semble pas que les écarts de développement aient été substanciellement réduits ${ }^{3}$. On

1. E. Moxon-Browne, Political change in Spain, op. cit.

2. C. Díaz López, «Center-periphery... », art. cité ; E. García de Enterria, «El futuro...", art. cité.

3. J. Cuadrado Roura, "Economic growth and regional disparities before and after the crisis : the Spanish case », dans G. Gorzelak (ed.), Regional dynamics of socio-economic change, Varsovie, University of Warsaw, Institute of Space Economy, 1988 ; J. Villaverde Castro, "A typology of the Spanish regions: a proposed approach », Revue d'économie régionale et urbaine, 4, 1988, p. 593-605; J.M. Rey Julia, "Les causalités circulaires et cumulatives. Le cas de l'Espagne : 1955-1987 », Revue d'économie méridionale, 157, 1992, p. 77-96. 


\section{Richard Balme et al.}

parle aujourd'hui des "quatre Espagnes ${ }^{1}$ : l'Espagne en crise (Pays Basque, Asturies, région Cantabrique), celle qui croît (façade méditerranéenne et Madrid), celle qui survit (Galice, Andalousie) et celle qui se dépeuple (Estrémadure, La Manche et centre du pays à l'exception de Madrid).

Face à ces inégalités territoriales de développement, la politique "régionale" est en Espagne une idée relativement ancienne. Elle a commencé à se développer dans les années 1960 sur le modèle français de la planification indicative mais en l'absence paradoxale de tout échelon politico-administratif régional. A la fin du franquisme, un coup d'arrêt est donné à la politique de territorialisation de la planification en raison des incertitudes économiques et politiques croissantes mais également du refus de créer des "pépinières de séparatisme " ${ }^{2}$. Durant la transition démocratique, la politique territoriale est mise entre parenthèses, du fait de l'accumulation des tensions politiques, de la réorganisation de l'appareil d'Etat et de l'absence de cadre juridique défini, et ne constitue pas une priorité gouvernementale. Elle ne resurgit véritablement qu'une fois le processus autonomique stabilisé, les communautés autonomes installées et dans la perspective de l'adhésion de l'Espagne à la CEE. Diverses mesures (fonds de compensation inter-territorial créé en 1984, loi sur les incitations régionales pour la correction des déséquilibres économiques inter-territoriaux, votée en 1985, obligeant chaque communauté autonome à se doter d'un plan de développement régional (PDR), l'ensemble des PDR étant synthétisés dans un PDR national soumis à la CEE pour bénéficier des aides du Fond européen de développement régional (FEDER, etc.) visent à rechercher une complémentarité des actions et des financements de l'Etat central, des communautés autonomes et de la CEE ${ }^{3}$.

On le voit, ces travaux s'inscrivent dans des perspectives différentes, plus complémentaires qu'opposées, et relèvent de disciplines académiques distinctes : droit, science politique, sociologie, économie pour l'essentiel. Aussi, compte tenu de cette intégration limitée des connaissances, est-il difficile de faire apparaître clairement des paradigmes dominants ou un déplacement significatif et véritable des problématiques en ce qui concerne l'étude, en Espagne, des relations entre le centre et la périphérie, appréhendées dans leur globalité. D'autant plus que ce champ de recherche est de constitution récente: les cultures scientifiques des auteurs espagnols, anglo-saxons et français ne sont pas identiques, et les études empiriques précises ne portant pas uniquement sur des régions phares

1. J. Alcaide Inchausti, «Las cuatro Españas económicas y la solidaridad regional », Papeles de economía española, 34, 1988, p. 62-81.

2. H. Richardson, Regional development policy and planning in Spain, Farnborough, Saxon House, 1975.

3. A. Fernández Díaz, "Política de planificación », dans L. Gamir (ed.), Política económica de España, Madrid, Alianza, 1990; G. Sáenz de Buruaga, "Política regional», dans L. Gamir (ed.), Politica económica de España, op. cit.; R. Tamames, Estructura económica de España, Madrid, Alianza, 1991; E. Urzainqui Miqueleiz, «Pouvoir central, communautés autonomes et politique régionale en Espagne ", Revue d'économie régionale et urbaine, 4, 1988, p. 707-719. 
telles que la Catalogne ou le Pays Basque sont encore peu nombreuses. Après une phase nécessaire de description privilégiant les références historiques et les normes juridiques ainsi que la généralité des processus, un approfondissement des connaissances et une explication des paradigmes paraissent donc devoir s'imposer pour progresser dans la compréhension et l'explication des phénomènes considérés.

\section{LA POLITIQUE RÉGIONALE DE LA COMMUNAUTÉ EUROPÉENNE}

La construction communautaire met en cause les politiques territoriales dans les Etats-membres, à un double titre. D'une part, le versant économique de l'intégration avec la signature de l'Acte unique en 1986 et l'entrée en vigueur du Grand Marché en 1993 affecte les échanges de capitaux et de main-d'œuvre, et tend à redistribuer les activités économiques selon de nouveaux modes de polarisation spatiale. D'autre part, l'aspect politique de l'intégration, c'est-à-dire l'élargissement des capacités d'initiative propres aux institutions européennes, se manifeste notamment par le développement de la politique régionale de la Communauté, qui exerce une influence de plus en plus déterminante sur la gestion territoriale des Etats-membres. Ceux-ci sont donc confrontés à des mutations spatio-économiques sans précédent dans leur intensité, que la construction communautaire ne fait qu'accélérer et amplifier, et à des cadres d'action publique à la fois débordés par ces mouvements, et contraints dans leurs modalités par les règlements ou les politiques communautaires. C'est ainsi la légitimité de l'intégration territoriale et de son traitement politique au niveau national qui est mise en cause ou reformulée sous l'effet de la construction européenne. Face à ces phénomènes, la littérature aborde trois types de questions que nous envisagerons successivement: quelle est l'évolution des disparités régionales dans la Communauté, et quels en sont les facteurs déterminants? Comment la politique régionale communautaire est-elle élaborée, et quelle est sa contribution à la construction européenne? Enfin, quels sont les effets de sa mise en cuvre dans les Etats-membres?

\section{L'ÉVOLUTION DES DISPARITÉS RÉGIONALES DANS LA COMMUNAUTÉ}

Le sujet est évidemment le terrain privilégié des économistes et des experts de la Commission, mais se révèle important en forgeant le cadre cognitif des politiques territoriales et de leur analyse. Les difficultés méthodologiques, notamment dans le choix des données et indicateurs, conduit le plus souvent les auteurs à la prudence et appelle des analyses critiques et nuancées ${ }^{1}$, mais il est néanmoins possible de dégager

1. P. Delfaux, «La perception des disparités régionales dans la Communauté économique européenne" ", Revue d'économie régionale et urbaine, 1, 1989, p. 41-70; P. Cheshire, «Problems of regional transformation and deindustrialization in the European Community ", dans L. Rodwin, H. Sazanami, Industrial change and economic transformation. The experience of Western Europe, Londres, Harper Collins Academic, 1991. 
quelques tendances essentielles. Les rapports périodiques établis par la Commission font état de l'importance et de la persistance des disparités entre les régions de la Communauté, au moins deux fois supérieures à celles des Etats-Unis d'Amérique. Sur le long terme, les écarts mesurés en termes de PIB, de revenu et de chômage suivent des mouvements oscillatoires. Les années 1960 sont une période de convergence entre les Etats-membres et les régions, prenant fin au milieu des années 1970. Sous l'effet de la récession, on a ensuite une phase de divergence qui ramène les disparités interrégionales aux niveaux du début des années 1970 , voire à des niveaux antérieurs, et, dans une troisième période, ce mouvement semble se stabiliser à partir de 1986. Parmi les facteurs expliquant ces disparités, il faut mentionner, outre les élargissements successifs, la conjonction entre le faible niveau de développement économique et l'évolution démographique des régions les plus pauvres, les avantages concurrentiels offerts aux investisseurs (transports, télécommunications, personnel qualifié, offre de formation, crédit et fiscalité) et les capacités d'innovation des entreprises conditionnant le potentiel de recherche et développement. Ces disparités prennent un caractère profond et durable : «Même en supposant la continuation de la récente évolution favorable, la convergence des Etats-membres plus faibles et des régions moins prospères vers la moyenne communautaire sera un processus de très longue haleine $"{ }^{1}$. Les observateurs soulignent l'aspect plus structurel que cyclique de ces mouvements, et l'incapacité des programmes publics à les contenir et, a fortiori, à les inverser, leur efficience étant finalement subordonnée à la conjonction de circonstances favorables qu'ils ne peuvent que conforter ${ }^{2}$. D'autres analysent l'aggravation des disparités provoquée par les politiques communautaires, notamment par la politique agricole $^{3}$ ou par la politique monétaire et tarifaire ${ }^{4}$, et leur contradiction avec la politique régionale dont elles annulent les effets ${ }^{5}$. Les recherches convergent pour constater la permanence et l'accentuation des clivages Nord-Sud mais aussi Est-Ouest, et surtout la polarisation du développement économique autour de la zone centrale de l'Europe ${ }^{6}$.

1. Commission des Communautés européennes, Les régions dans les années 1990 , Quatrième rapport sur la situation et l'évolution socio-économiques des régions de la Communauté, Bruxelles, 1991 , p. 18.

2. L. Rodwin, "European industrial change and regional economic transformation: an overview of recent experience ", dans L. Rodwin, H. Sazanami, Industrial change..., op. cit., p. 3-38.

3. E. Gasperini, "Intégration européenne et accroissement des disparités régionales. $\mathrm{La}$ politique de la Communauté économique européenne en question ", Economie et société, 31 , février 1989 , p. $129-159$ (hors série); D. Gabdin, "Quelle politique régionale pour la Communauté économique européenne?", Revue du Marché commun, 314, février 1988, p. 68-74; D. Strijker, J. de Veer, "Agriculture », dans W. Molle, R. Cappelin, Regional impact of community policies in Europe, Aldershot, Gower, 1988.

4. P. Cheshire, R.P. Camagni, J.-P. de Gaudemar, J.R. Cuadrado Rura, « 1957 to 1992: moving toward a Europe of regions and regional policy », dans L. Rodwin, H. Sazanami, Industrial change..., op. cit., p. 268-300.

5. J. Grote, "Diseconomies in space: traditional sectoral policies of the EC, the European technology community and their effects on regional disparities », Regional Policies and Politics, juin 1992.

6. R. Brunet, Les villes européennes, Paris, La Documentation française, 1989 ; 
L'ÉLABORATION DE LA POLTTIQUE RÉGIONALE DE LA COMMUNAUTÉ ET L'INTÉGRATION EUROPÉENNE

Ici, le débat est sans doute moins complexe puisqu'il s'agit principalement de suivre les développements juridiques et institutionnels de la politique régionale de la CEE. Cette politique ne s'est constituée que progressivement ${ }^{1}$ puisque la création du FEDER date de 1975, et qu'elle est longtemps restée complémentaire par rapport aux politiques nationales avant de s'autonomiser peu à peu ${ }^{2}$. Plusieurs points doivent être soulignés. En premier lieu, c'est l'intégration européenne, avec les élargissements successifs et les perspectives du marché puis, plus récemment, de la monnaie unique, qui constitue le moteur de la politique régionale communautaire. Elle est surtout un terrain de concessions mutuelles qui en fait un moyen beaucoup plus qu'un objectif de l'intégration, supposée pour les représentants des Etats-membres produire d'elle-même la convergence économique sur le long terme. Certains notent d'ailleurs que les indicateurs retenus à l'origine dans l'estimation des disparités régionales, à savoir principalement le PIB par habitant ou par actif, reflètent une conception de la convergence basée bien davantage sur le développement économique que sur les transferts de revenus ${ }^{3}$. On peut lire dans la réforme des fonds structurels de 1987, et a fortiori dans la difficile négociation du «deuxième paquet Delors» consécutive au traité de Maastricht, un changement de philosophie de la politique régionale communautaire, impulsé par la présidence de la Commission, lui assignant un rôle moins compensatoire et plus directement actif dans la convergence. Les perspectives de nouveaux élargissements vers l'Est donnent évidemment toute son actualité à cet aggiornamento. Mais elles n'infirment pas la vision dominante de la DG XVI (Direction générale de la politique régionale), plus sensible à une «conception nordiste du développement », sinon endogène, reposant en tout cas sur les acteurs privés, qu'à l'aménagement du territoire sur le modèle français, italien ou espagnol ${ }^{4}$. Il faut également souligner que les politiques nationales ou régionales de subventions industrielles dans les Etats-membres entrent fréquemment en conflit avec la politique de concurrence de la Communauté, mise en œuvre par la DG IV, qui les prive ainsi d'un outil de gestion territoriale important, tout en les incitant, par le caractère conditionnel des aides régionales européennes, à intervenir selon des modalités différentes. Ce processus normatif, sanctionné par l'application

J.R. Sortia, C.H. Vandermotten, J. Vandalaer, Atlas économique de l'Europe, Bruxelles, SRBG, Université libre de Bruxelles, 1986.

1. Y. Doutriaux, La politique régionale de la CEE, Paris, PUF, 1991.

2. P. Pascallon, "Historique de la politique communautaire d'aménagement du territoire ", Revue d'économie régionale et urbaine, 5, 1990, p. 681-693.

3. J. Mawson, M.R. Martins, J.T. Gibney, "The development of the European Community regional policy ", dans M. Keating, B. Jones, Regions in the European Community, Oxford, Clarendon Press, 1985, p. 20-59.

4. F. Celimène, C. Lacour, "La réforme des fonds structurels européens : éléments d'une théorie du développement régional communautaire », Revue d'économie régionale et urbaine, 2, 1991, p. 183-219. 
Richard Balme et al.

des règlements et du droit communautaire et par l'accès au soutien financier de Bruxelles, semble conduire à une modernisation et à une convergence des politiques territoriales dans les Etats-membres.

\section{LES EFFETS DE LA MISE EN CEUVRE : VERS L'EUROPE DES RÉGIONS?}

Si le développement des politiques de la Communauté et leur mise en œuvre affectent indéniablement les relations centre-périphérie dans les Etats-membres, les effets globaux en sont relativement difficiles à cerner. On peut certes envisager des scénarios d'affaiblissement ou de consolidation des administrations et des gouvernements centraux dans leur position intermédiaire entre les régions ou les gouvernements locaux et les institutions européennes. L'observation fait néanmoins apparaître une réalité plus complexe, variant d'un pays à l'autre, souvent traversée de tendances contradictoires, et dont la forme définitive est encore incertaine.

Les analyses s'accordent sur l'importance, dans l'ensemble des pays, des administrations centrales dans la mise en ouvre des fonds structurels, pour des raisons de capacités administratives de la Commission et des régions, lorsqu'elles existent. Les niveaux centraux de gouvernement ne peuvent donc pas être considérés comme marginalisés par la politique régionale communautaire, et gagnent parfois en influence sur les régions, en Italie, en Espagne ${ }^{1}$ et, au moins jusqu'à la mise en œuvre de la réforme des fonds structurels, en Allemagne ${ }^{2}$. Les politiques de réduction des disparités régionales des Etats-membres sont orientées, parfois suscitées ou minimalement maintenues, comme en Grande-Bretagne, sous l'effet des programmes communautaires. Les régions étendent également leurs prérogatives, puisque les Länder allemands (comme les régions belges) sont associés aux instances fédérales dans l'élaboration de la politique communautaire. Elles sont cependant en position très différente face aux politiques communautaires ${ }^{3}$. Si les financements européens sont toujours les bienvenus en période de récession et de retrait des politiques nationales d'aménagement, les régions les plus différenciées ont une meilleure influence sur Bruxelles, en raison de leurs compétences juridiques et organisationnelles. Mais elles sont aussi plus sensibles aux contraintes imposées par la législation et les politiques communautaires qui interfèrent davantage avec les politiques locales lorsque celles-ci sont plus développées. Les Länder allemands sont réticents devant les exigences posées par la Commission pour soutenir leurs propres politiques d'aménagement et la lourdeur des procédures qu'elle inflige ${ }^{4}$. Au total,

1. F. Morata, «La implementación regional de las políticas comunitarias », Quaderns de Treball de l'Institut Universitari d'Estudis Europeus, 7, 1991, 98 p.

2. P. Malanczuk, «Les politiques communautaires et les Länder en République fédérale d'Allemagne », Revue française d'administration publique, 34, 1985, p. 283-302; H.G. Gestenlauer, "German Länder in the Eurropean Community », dans M. Keating, B. Jones, Regions in the European Community, Oxford, Clarendon Press, 1985, p. 173-190.

3. M. Keating, B. Jones, Regions in the European Community, Oxford, Clarendon Press, 1985.

4. J.F. Anderson, "Skeptical reflections on a Europe of regions : Britain, Germany and the ERDF ", Journal of Public Policy, 10 (4), 1990, p. 417-447. 
les effets des politiques communautaires, en prenant pour cadre des systèmes politico-administratifs différenciés, sont certainement dominés par la diversité. A titre d'hypothèse, on peut envisager qu'ils produisent un mouvement de convergence limitée, encadrant l'autonomie régionale là où elle est la plus forte et la confortant là où elle est la plus faible, conduisant dans tous les cas à une intégration accrue entre le centre et la périphérie ${ }^{1}$.

Les recherches dans ce domaine s'accordent également pour souligner l'importance de la coopération trans-frontalière et interrégionale, et le développement de groupes de pression trans-nationaux défendant les intérêts régionaux auprès des institutions communautaires à Bruxelles ou Strasbourg ${ }^{2}$. On ne peut ici que mentionner l'explosion du nombre de bureaux ouverts à Bruxelles par les régions ou les gouvernements locaux, quelquefois sous forme groupée, et la création par la Commission en 1988, en réponse aux appels du Parlement européen, du Conseil consultatif des autorités régionales et locales, puis du Comité des régions prévu par le traité de Maastricht (art. 198 A), qui peut être officiellement consulté au sujet de la politique régionale. Dans l'hypothèse forte d'une Europe des régions concurrençant directement les niveaux centraux de gouvernement, on est tenté d'y voir la préfiguration d'un Sénat européen intégrant les régions. Si une telle éventualité reste soumise à trop de difficultés institutionnelles (les disparités de statut des régions en Europe) et politiques (les résistances du Conseil) pour être plausible à court et moyen terme, ces tendances d'évolution sont néanmoins significatives. Le changement d'échelle des échanges et des interdépendances économiques remet en cause les régulations politiques forgées dans le cadre des Etats-nations, et s'accompagne d'une recomposition des territoires et des réseaux de politiques publiques ${ }^{3}$. Ceux-ci sont aujourd'hui marqués par trois caractères importants: ils sont situés à un niveau de mésogouvernement, intermédiaire entre les institutions centrales et les gouvernements strictement locaux, et placent souvent la région en position nodale. Ils sont transversaux, associant de plus en plus fréquemment les gouvernements locaux dans des procédures de coopération. Ils sont enfin soumis à un processus d'internationalisation, débordant les frontières étatiques et contribuant au système complexe et inachevé de relations entre les institutions communautaires et les intérêts organisés. Leur étude s'avère donc importante pour la compréhension des évolutions récentes des gouvernements locaux, mais aussi pour aborder les phénomènes d'intégration et de désintégration territoriale dans le cadre des Etats-

1. R. Balme, "EEC regional policies and policy networks in the member-States », contribution à l'European Consortium for Political Research, Joint Session of Workshops, Essex, 1991 .

2. M. Keating, B. Jones, Regions in the European Community, nouvelle éd. à paraître.

3. M. Keating, "The continental meso. Regions in the European Community ", dans L.J. Sharpe, Between centre and locality. Meso-government in Europe, Londres, Sage, 1991 ; W. Streeck, P.C. Schmitter, "From national corporatism to transnational pluralism : organized interests in the Single European Market », Politics and Society, 19 (2), 1991, p. 133-164. 
Richard Balme et al.

nations, et enfin pour interpréter la dynamique des institutions et du système politique européen.

Richard Balme (chargé de recherche au Centre d'étude et de recherche sur la vie locale (CERVL), Institut d'études politiques de Bordeaux) est l'auteur de "French regions facing European integration", dans B. Jones, $M$. Keating, Regions in the EEC, Oxford, Clarendon Press, 1993. Il travaille sur la comparaison des politiques publiques et sur les dimensions territoriales de l'intégration européenne.

Philippe Garraud (chargé de recherche CNRS, CERVL) est l'auteur de Profession: homme politique, Paris, l'Harmattan, 1989. Il travaille sur le métier politique, les politiques de lutte contre le chômage et, plus récemment, sur la politique espagnole.

Vincent Hoffmann-Martinot (chargé de recherche CNRS, CERVL) est l'auteur de "Grüne and Verts. Two faces of European ecologism ", West European Politics, octobre 1991; "La participation aux élections municipales dans les villes françaises", Revue française de science politique, 42 (1), février 1992. Il travaille sur la comparaison des systèmes politiques locaux des pays industrialisés.

Evelyne Ritaine (chargé de recherche CERVL) est l'auteur de "Territoire: espace du jeu politique ", Quaderni, 13-14, 1991. Elle travaille sur la territorialisation du politique en Italie et sur les médiations sociopolitiques en Europe du sud.

Laurence Bonnet et Stéphane Le May, tous deux doctorants, sont allocataires de recherche au CERVL (CERVL, Institut d'études politiques de Bordeaux, BP 101, 33405 Talence).

En raison de la publication intégrale des «Travaux universitaires inédits », la rubrique « Revue des revues » est renvoyée au prochain numéro. 